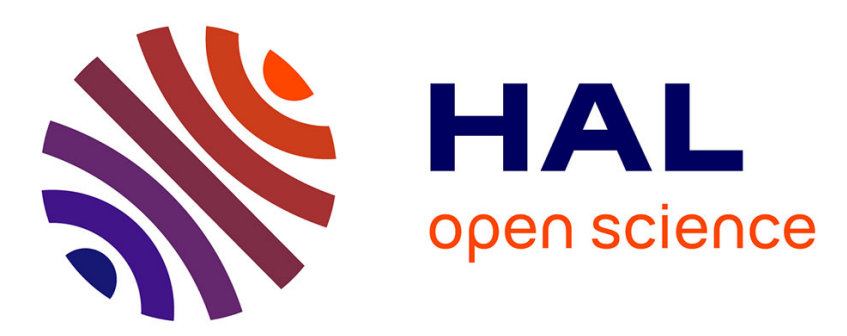

\title{
Fault Detection, Isolation and Quantification from Gaussian Residuals with Application to Structural Damage Diagnosis
}

\author{
Michael Döhler, Laurent Mevel, Qinghua Zhang
}

\section{- To cite this version:}

Michael Döhler, Laurent Mevel, Qinghua Zhang. Fault Detection, Isolation and Quantification from Gaussian Residuals with Application to Structural Damage Diagnosis. Annual Reviews in Control, 2016, 42, pp.244-256. 10.1016/j.arcontrol.2016.08.002 . hal-01376804

\section{HAL Id: hal-01376804 \\ https://inria.hal.science/hal-01376804}

Submitted on 5 Oct 2016

HAL is a multi-disciplinary open access archive for the deposit and dissemination of scientific research documents, whether they are published or not. The documents may come from teaching and research institutions in France or abroad, or from public or private research centers.
L'archive ouverte pluridisciplinaire HAL, est destinée au dépôt et à la diffusion de documents scientifiques de niveau recherche, publiés ou non, émanant des établissements d'enseignement et de recherche français ou étrangers, des laboratoires publics ou privés. 


\title{
Fault Detection, Isolation and Quantification from Gaussian Residuals with Application to Structural Damage Diagnosis ${ }^{\text {th }}$
}

\author{
Michael Döhler*, Laurent Mevel, Qinghua Zhang \\ Inria / IFSTTAR, I4S, Campus de Beaulieu, 35042 Rennes, France
}

\begin{abstract}
Despite the general acknowledgment in the Fault Detection and Isolation (FDI) literature that FDI are typically accomplished in two steps, namely residual generation and residual evaluation, the second step is by far less studied than the first one. This paper investigates the residual evaluation method based on the local approach to change detection and on statistical tests. The local approach has the remarkable ability of transforming quite general residuals with unknown or non Gaussian probability distributions into a standard Gaussian framework, thanks to a central limit theorem. In this paper, the ability of the local approach for fault quantification will be exhibited, whereas previously it was only presented for fault detection and isolation. The numerical computation of statistical tests in the Gaussian framework will also be revisited to improve numerical efficiency. An example of vibration-based structural damage diagnosis will be presented to motivate the study and to illustrate the performance of the proposed method.
\end{abstract}

Keywords: Fault detection and isolation, Fault quantification, Local approach, Residual evaluation, Hypothesis tests, Minmax tests, Structural damage diagnosis

\section{Introduction}

The complexity of modern engineering systems grows with the requirements on economic performance and on quality of production or service, while subject to safety and environmental constraints. In this trend, fault diagnosis is becoming an integrated functionality of more and more systems, from industrial processes to consumer products. Model-based methods for Fault Detection and Isolation (FDI) have been widely studied since several decades (Basseville and Nikiforov, 1993; Gertler, 1998; Chen and Patton, 1999; Simani et al., 2003; Korbicz, 2004; Isermann, 2006; Ding, 2008; Blanke et al., 2015). In this research field, most studied systems are dynamic, in the sense that the current outputs of a system depend not only on the current inputs, but also on the history of the system. Such systems are typically described by differential equations. The resulting methods for FDI typically follow two steps, namely residual generation and residual evaluation (Hwang et al., 2010).

The purpose of residual generation is to generate, through the processing of raw sensor signals, some residual signals that are typically close to zero in the fault-free case and significantly different from zero in the faulty case. Because of the dynamic nature of the considered systems and the lack of sensors for full

\footnotetext{
The material in this paper was partially presented at the 19th IFAC World Congress (IFAC'14), August 24-29, 2014, Cape Town, South Africa, and at the 9th IFAC Symposium on Fault Detection, Supervision and Safety for Technical Processes (SAFEPROCESS'15), September 2-4, 2015, Paris, France.

*Corresponding author. Tel: +332998422 25.

Email addresses: michael.doehler@inria.fr (Michael Döhler), laurent.mevel@inria.fr (Laurent Mevel), qinghua.zhang@inria.fr (Qinghua Zhang)
}

state measurement, residual generation is by far not a trivial task. Most methods for residual generation are based on fault detection filters (Massoumnia, 1986; Edelmayer et al., 1997; Chen and Speyer, 2000; Zhong et al., 2010), state observers (Hammouri et al., 1998; Besançon, 2003; Xu and Zhang, 2004; Hammouri and Tmar, 2010), and parity relations (Medvedev, 1995; Gertler, 1997). Essentially these methods compensate the lack of sensors by taking into account the dynamic nature of the considered system. Some methods lead to residuals focusing on part of the possible faults for the purpose of fault isolation (Gertler, 1998; Blanke et al., 2015). Some other methods design robust residuals, which are fully or partly decoupled from unknown disturbances (Chen and Patton, 1999; Henry et al., 2014).

Due to measurement noises and modeling errors, residuals are not perfectly zero in the fault-free case. Residual evaluation is thus not a trivial task, either. Despite the general acknowledgment of the two steps in FDI methods and the importance of each step, most publications focus on the residual generation step. The major methods for residual evaluation are based on the theory of statistics (Basseville and Nikiforov, 1993; Basseville, 1997) by assuming random uncertainty models, whereas some other methods rely on interval computations by assuming bounded uncertainties (Puig, 2010; Raka and Combastel, 2013).

Statistical methods for residual evaluation usually start by assuming some probability distribution of measurement and modeling errors, typically Gaussian, or sometimes even directly making such assumptions about the residuals. Such an approach is more for theoretic convenience than for physical reasons. To relax these assumptions, the local approach to change 
detection (Benveniste et al., 1987) has the remarkable ability of transforming quite general residuals with unknown probability distributions into a standard Gaussian framework, thanks to a Central Limit Theorem (CLT). The local approach is thus a powerful tool for statistical evaluation of residuals. The essential assumption behind the local approach is that the considered faults are characterized by small parametric changes. It is thus particularly suitable for incipient fault diagnosis. The meaning of "small" will be explained later in this paper when some details of this approach are presented. While many FDI methods concern additive faults, e.g. (Dong and Verhaegen, 2009; Dong et al., 2012), our approach assumes a more general description of incipient faults linked to small parameter changes. While the detection and diagnosis of incipient faults from random noise-corrupted data are considered in this paper, other aspects of incipient faults have been studied in the literature, for instance, the dynamics of observer based residuals in (Escobet et al., 2014), and the variabilities of data covariance eigenvalues in (Harmouche et al., 2014). Concerning fault estimation or quantification, some methods are focused on additive sensor faults, e.g. the sliding mode observer-based method (Tan and Edwards, 2002). Other methods are based on parameter estimation or system identification techniques (Isermann, 2005). These methods do not consider the particular case of incipient fault quantification from noise-corrupted sensor data, which is addressed in the present paper.

The purpose of the present paper is twofold. First, the ability of the local approach for fault quantification will be exhibited, whereas previously this approach was only presented for fault detection and isolation. In this way, a common framework for fault detection, isolation and quantification is achieved. Second, the numerical computation of statistical tests in the Gaussian framework will be revisited, in order to improve the numerical efficiency of the residual evaluation method based on the local approach.

To motivate the results presented in this paper, an example of vibration-based damage diagnosis in the context of Structural Health Monitoring (SHM) will be presented in detail. SHM consists in detecting and characterizing damages in engineering structures, such as civil, aeronautical or mechanical structures from measurements (Farrar and Worden, 2007; Brownjohn, 2007). We consider the monitored structure as a linear time-invariant system subject to state and measurement noise. Usually, measurements are output-only, since the inputs are typically ambient excitations (like traffic or wind), which cannot be measured with a sufficient accuracy for vibration analysis. Damage diagnosis consists of several levels of increasing difficulty, beginning with damage detection (corresponding to fault detection), damage localization (fault isolation) and damage quantification (fault quantification). The faults - structural damages - affect the dynamic properties of the monitored structure, inducing changes in the modal parameters (natural frequencies, damping ratios, mode shapes), or in the equivalent eigenstructure representation of the linear system (eigenvalues and observed eigenvectors). It is of interest to diagnose damages at an early stage before they grow to dangerous extents. Damage detection thus amounts to detecting small changes in the eigenstructure of the monitored structure. Damage localization usually requires a finite element (FE) model of the structure and links changes in the eigenstructure to changes in the FE parameters. The location of the damage then corresponds to regions in the model where the respective FE parameters have changed. Damage quantification corresponds to estimation of the parameter change. Together with an appropriate residual function, it is shown how these diagnosis problems can be solved with the techniques developed in this paper.

In this context, subspace-based residuals and detection tests have been proposed in (Basseville et al., 2000; Döhler and Mevel, 2013; Döhler et al., 2014b) with field applications, e.g. in (Döhler et al., 2014a). For damage localization, it can be decided with minmax or sensitivity approaches which components in the FE parameter set have changed. While sensitivity tests have been used for damage localization in (Basseville et al., 2004; Balmès et al., 2008), we show in this paper that minmax tests have more appropriate properties and are better suited for the damage localization problem when the normalized parameter sensitivities are not orthogonal. In the context of vibration monitoring, minmax approaches have also been applied to damping monitoring (Zouari et al., 2009) or damage detection with temperature rejection (Balmès et al., 2009). While these studies have been focused on FDI, it has never been attempted to quantify the absolute change in the faulty parameter components.

This paper is organized as follows. In Section 2, the problems of fault detection, isolation and quantification in the context of the asymptotic local approach to change detection are introduced. The appropriate hypothesis tests for fault detection and isolation are introduced in Section 3. Schemes for an efficient numerical computation of these tests are developped in Section 4. In Section 5, fault quantification is addressed and applied to structural damage diagnosis in Section 6. Numerical applications of the proposed methods are presented in Section 7.

\section{Problem statement}

Let a system be characterized by a parameter vector $\theta$. Assume that measurements $\mathcal{y}_{N}=\left\{Y_{1}, \ldots, Y_{N}\right\}$ of length $N$ collected from the system are the realization of an asymptotically stationary stochastic process depending on the parameter vector $\theta$.

We consider the problems of detecting, isolating and quantifying faults, which are deviations of the considered system from the nominal behavior characterized by some nominal parameter value $\theta_{0}$. In particular, small deviations are considered for the diagnosis of incipient faults. With the asymptotic local approach to change detection (Le Cam, 1956, 1986; Benveniste et al., 1987), the fault diagnosis problem for a parametrized stochastic process is transformed into monitoring the mean of a Gaussian residual vector. The local approach assumes the close hypotheses

$$
\begin{array}{ll}
\mathbf{H}_{0}: \theta=\theta_{0} & \text { (reference system) }, \\
\mathbf{H}_{1}: \theta=\theta_{0}+\delta / \sqrt{N} & \text { (faulty system), }
\end{array}
$$


where vector $\delta$ is unknown but fixed. This corresponds to a very general description of a fault linked to any small change in the system parameter $\theta$. The system dynamics can be parametrized by $\theta$ in a complex manner, and generally parameter changes affect measured outputs in a non-additive manner (see example in Section 6). With this framework, very small changes in $\theta$ can be detected if the number of measurements $N$ is large enough, by linking this change to $1 / \sqrt{N}$. Confronting the measurements to the nominal system parameter, a residual vector $\zeta\left(\theta_{0}, \mathcal{Y}_{N}\right) \stackrel{\text { def }}{=} \frac{1}{\sqrt{N}} \sum_{k=1}^{N} h\left(\theta_{0}, Y_{k}\right)$ is designed, through a socalled primary residual $h\left(\theta_{0}, Y_{k}\right)$ satisfying some basic conditions (Benveniste et al., 1987). First, the identifiability property

$$
\mathbf{E}_{\theta} h\left(\theta_{0}, Y_{k}\right)=0 \text { iff } \theta=\theta_{0}
$$

is assumed, where $\mathbf{E}_{\theta}$ denotes the expectation over realizations of $Y_{k}$ under the true system parameter $\theta$. Second, differentiability with respect to $\theta$ is assumed in a neighborhood of $\theta_{0}$, and furthermore the process $h\left(\theta_{0}, Y_{k}\right)$ satisfies some mild mixing conditions. Then, the main result of the local approach based on assumptions (1) is a central limit theorem (CLT) ensuring that

$$
\zeta\left(\theta_{0}, \mathcal{Y}_{N}\right) \stackrel{d}{\longrightarrow} \begin{cases}\mathcal{N}(0, \Sigma) & \text { under } \mathbf{H}_{0} \\ \mathcal{N}(\mathcal{J} \delta, \Sigma) & \text { under } \mathbf{H}_{1}\end{cases}
$$

for $N \rightarrow \infty$, where $\mathcal{J}$ and $\Sigma$ are the asymptotic sensitivity at $\theta_{0}$ and covariance of the residual, respectively. For example, as recalled in Section 6, an appropriate residual vector for vibration monitoring has been proposed based on subspace properties (Basseville et al., 2000; Döhler and Mevel, 2013; Döhler et al., 2014b). Note that the modeling of the change in $\theta$ as $\delta / \sqrt{N}$ in hypothesis (1) has been introduced to obtain CLT (2) with constant mean $\mathcal{J} \delta$ under $\mathbf{H}_{1}$, resulting from a first-order Taylor expansion of the residual.

In detail, we consider the following diagnosis problems in this framework:

- Fault detection, i.e. change detection in $\theta$, corresponds to deciding if $\delta \neq 0$;

- Fault isolation, i.e. deciding which parameters in $\theta$ are faulty, corresponds to deciding which components of $\delta$ are non-zero;

- Fault quantification corresponds to estimating the change $\left(\theta-\theta_{0}\right)$ in the faulty components.

Based on the asymptotic residual distribution in (2), the considered diagnosis problems can be solved in a standard Gaussian framework with statistical tests and parameter estimation methods, as described in the following sections.

\section{Basic fault detection and isolation tests}

In this section, we recall hypotheses tests for a parametrized Gaussian residual vector $\zeta \in \mathbb{R}^{h}$ between the two hypotheses

$$
\zeta \sim \begin{cases}\mathcal{N}(0, \Sigma) & \text { Hypothesis } \mathbf{H}_{0} \\ \mathcal{N}(\mathcal{J} \delta, \Sigma) & \text { Hypothesis } \mathbf{H}_{1}\end{cases}
$$

where $\delta \in \mathbb{R}^{l}$ is an unknown vector with $\delta \neq 0$ under $\mathbf{H}_{1}$, the sensitivity matrix $\mathcal{J} \in \mathbb{R}^{h \times l}$ has full column rank and the covariance matrix $\Sigma \in \mathbb{R}^{h \times h}$ is positive definite. It is assumed that the sensitivity and covariance matrices $\mathcal{J}$ and $\Sigma$ are known or estimated somehow in practice. Many methods have been proposed in the literature for statistical fault detection (decide if $\delta=0$ or $\delta \neq 0$ ) and isolation (decide which components of vector $\delta$ are non-zero) in the framework of such a Gaussian residual vector $\zeta$ (Basseville and Nikiforov, 1993; Basseville, 1997). In the following, we recall the appropriate generalized likelihood ratio (GLR) tests.

\subsection{Fault detection: global test}

The global test is intended to make a decision between $\delta=0$ and $\delta \neq 0$ based on residual vector $\zeta$ in (3). The GLR test applied to this problem leads to the test statistic (Basseville, 1997)

$$
t_{\text {global }}=\zeta^{T} \Sigma^{-1} \mathcal{J}\left(\mathcal{J}^{T} \Sigma^{-1} \mathcal{J}\right)^{-1} \mathcal{J}^{T} \Sigma^{-1} \zeta
$$

which follows a $\chi^{2}$ distribution with $l$ degrees of freedom and the non-centrality parameter $\delta^{T} F \delta$, where $F=\mathcal{J}^{T} \Sigma^{-1} \mathcal{J}$ is the Fisher information matrix. To decide between $\mathbf{H}_{0}$ and $\mathbf{H}_{1}$, the test variable $t_{\text {global }}$ is compared to a threshold. Typically the threshold is chosen so that the probability of false alarms is below some chosen level. In theory this choice can be made according to the $\chi^{2}$ distribution of $t_{\text {global }}$ from the reference system.

\subsection{Fault isolation: sensitivity and minmax tests}

For the problem of fault isolation it has to be decided which components of vector $\delta$ are non-zero, which can be done by testing each component (or group of components) of $\delta$ separately. Thus, consider different partitions of the vector $\delta$ into two subvectors, where one of the subvectors is tested. Without loss of generality, let this partition be

$$
\delta=\left[\begin{array}{l}
\delta_{a} \\
\delta_{b}
\end{array}\right]
$$

For fault isolation, $\delta_{a}=0$ is tested against $\delta_{a} \neq 0$ for each partition. Let the sensitivity and Fisher information matrices be partitioned accordingly as

$$
\mathcal{J}=\left[\begin{array}{ll}
\mathcal{J}_{a} & \mathcal{J}_{b}
\end{array}\right], F=\left[\begin{array}{ll}
F_{a a} & F_{a b} \\
F_{b a} & F_{b b}
\end{array}\right]=\left[\begin{array}{cc}
\mathcal{J}_{a}^{T} \Sigma^{-1} \mathcal{J}_{a} & \mathcal{J}_{a}^{T} \Sigma^{-1} \mathcal{J}_{b} \\
\mathcal{J}_{b}^{T} \Sigma^{-1} \mathcal{J}_{a} & \mathcal{J}_{b}^{T} \Sigma^{-1} \mathcal{J}_{b}
\end{array}\right]
$$

In the following subsections, two isolation tests are recalled.

\subsubsection{Sensitivity test}

The simplest possibility for testing $\delta_{a}=0$ against $\delta_{a} \neq 0$ is to assume $\delta_{b}=0$ and thus $\zeta \sim \mathcal{N}\left(\mathcal{J}_{a} \delta_{a}, \Sigma\right)$. The corresponding GLR test statistic writes as (Basseville, 1997)

$$
t_{\text {sens }}=\zeta^{T} \Sigma^{-1} \mathcal{J}_{a}\left(\mathcal{J}_{a}^{T} \Sigma^{-1} \mathcal{J}_{a}\right)^{-1} \mathcal{J}_{a}^{T} \Sigma^{-1} \zeta
$$

which is called sensitivity test. The test variable $t_{\text {sens }}$ is $\chi^{2}$ distributed with $\operatorname{dim}\left(\delta_{a}\right)$ degrees of freedom and non-centrality 
parameter $\delta_{a}^{T} F_{a a} \delta_{a}$. For a decision, the test variable is compared to a threshold, which is obtained for a given probability of false alarms according to the $\chi^{2}$ distribution of the reference system.

This holds if $\delta_{b}=0$ is actually true. If the assumption $\delta_{b}=0$ does not hold, the non-centrality parameter of $t_{\text {sens }}$ writes as

$$
\delta_{a}^{T} F_{a a} \delta_{a}+2 \delta_{a}^{T} F_{a b} \delta_{b}+\delta_{b}^{T} F_{b a} F_{a a}^{-1} F_{a b} \delta_{b},
$$

which follows from the properties of $\tilde{\zeta} \stackrel{\text { def }}{=}$ $\left(\mathcal{J}_{a}^{T} \Sigma^{-1} \mathcal{J}_{a}\right)^{-1 / 2} \mathcal{J}_{a}^{T} \Sigma^{-1} \zeta . \quad$ Since $\zeta \sim \mathcal{N}(\mathcal{J} \delta, \Sigma)$ and $\mathcal{J} \delta=\mathcal{J}_{a} \delta_{a}+\mathcal{J}_{b} \delta_{b}$,

$$
\tilde{\zeta} \sim \mathcal{N}\left(F_{a a}^{1 / 2} \delta_{a}+F_{a a}^{-1 / 2} F_{a b} \delta_{b}, I\right)
$$

using (5). From this normal distribution, the $\chi^{2}$ distribution of $t_{\text {sens }}=\tilde{\zeta}^{T} \tilde{\zeta}$ follows immediately with non-centrality parame$\operatorname{ter}(7)$.

\subsubsection{Minmax test}

Instead of assuming $\delta_{b}=0$, the variable $\delta_{b}$ is replaced by its least favorable value for a decision about $\delta_{a}$, leading to the minmax test (Basseville, 1997) as presented in the following. Define the partial residuals as

$$
\begin{aligned}
& \zeta_{a} \stackrel{\text { def }}{=} \mathcal{J}_{a}^{T} \Sigma^{-1} \zeta, \\
& \zeta_{b} \stackrel{\text { def }}{=} \mathcal{J}_{b}^{T} \Sigma^{-1} \zeta,
\end{aligned}
$$

the robust residual as

$$
\zeta_{a}^{*} \stackrel{\text { def }}{=} \zeta_{a}-F_{a b} F_{b b}^{-1} \zeta_{b}
$$

and

$$
F_{a}^{*} \stackrel{\text { def }}{=} F_{a a}-F_{a b} F_{b b}^{-1} F_{b a} .
$$

Then, the mean of the robust residual $\zeta_{a}^{*}$ is sensitive to changes $\delta_{a}$, but blind to $\delta_{b}$, and it holds

$$
\zeta_{a}^{*} \sim \mathcal{N}\left(F_{a}^{*} \delta_{a}, F_{a}^{*}\right)
$$

The corresponding GLR test statistic for $\delta_{a}=0$ against $\delta_{a} \neq 0$ writes as

$$
t_{\mathrm{mm}}=\zeta_{a}^{* T} F_{a}^{*-1} \zeta_{a}^{*}
$$

which is called minmax test. The test variable $t_{\mathrm{mm}}$ is $\chi^{2}$ distributed with $\operatorname{dim}\left(\delta_{a}\right)$ degrees of freedom and non-centrality parameter $\delta_{a}^{T} F_{a}^{*} \delta_{a}$, independently of $\delta_{b}$. For a decision, the test variable is compared to a threshold, which is obtained for a given probability of false alarms from the $\chi^{2}$ distribution of the reference system. Note that the invertibility of all matrices in the computation is guaranteed, when $\mathcal{J}$ has full column rank and $\Sigma$ is positive definite as assumed.

\subsubsection{Discussion}

The sensitivity and the minmax approaches differ by their assumption on $\delta_{b}$, where the minmax approach provides a more general setting. The sensitivity test assumes $\delta_{b}=0$, which may lead incoherent results if in reality $\delta_{b} \neq 0$, while the minmax test is not influenced by the true value of $\delta_{b}$. Only in the special case $F_{a b}=0$, i.e. if the normalized sensitivities $\Sigma^{-1 / 2} \mathcal{J}_{a}$ and $\Sigma^{-1 / 2} \mathcal{J}_{b}$ are orthogonal to each other, the sensitivity test statistic is independent of $\delta_{b}$, as can be seen in (7). In general, the sensitivity approach may have an unexpected behavior if the assumption $\delta_{b}=0$ is not satisfied. Thus, in a general setting the minmax should be preferred to the sensitivity test, while in related previous works (Basseville et al., 2004; Balmès et al., 2008) only the sensitivity approach was considered.

\section{Numerically efficient computation of the test statistics}

The tests from the previous section require a number of matrix inversions, which may be numerically critical due to possible ill-conditioning of the sensitivity and covariance matrices. In this section, we present numerical computations of the test statistics, where the number of matrix inversions is kept to a minimum. Instead, properties of the QR decomposition are efficiently used. Based on this development, we furthermore propose a fast computation scheme for multiple minmax tests, where different subvectors of $\delta$ are tested.

\subsection{Inverting the covariance matrix $\Sigma$}

Common to all tests is the inversion of the covariance matrix $\Sigma$. In the efficient computation method presented below, it turns out that only the matrix square root of the inverse, $\Sigma^{-1 / 2}$ will be needed with the property

$$
\Sigma^{-1}=\left(\Sigma^{-1 / 2}\right)^{T} \Sigma^{-1 / 2} .
$$

Its computation is usually made through the singular value decomposition (SVD) of $\Sigma$. By assumption, matrix $\Sigma$ is positive definite. From its SVD

$$
\Sigma=U \Lambda U^{T}
$$

where $U$ is an orthogonal matrix $\left(U^{T} U=I\right)$ and $\Lambda=$ $\operatorname{diag}\left(\lambda_{1}, \ldots, \lambda_{h}\right)$ with the singular values $\lambda_{1}, \ldots, \lambda_{h}$ in decreasing order, the matrix $\Sigma^{-1 / 2}$ can be obtained from

$$
\Sigma^{-1 / 2}=\Lambda^{-1 / 2} U^{T} \in \mathbb{R}^{h \times h},
$$

where $\Lambda^{-1 / 2}=\operatorname{diag}\left(\lambda_{1}^{-1 / 2}, \ldots, \lambda_{h}^{-1 / 2}\right)$, fulfilling (13). In order to prevent large numerical errors when $\Sigma$ is badly conditioned, $\lambda_{i}^{-1 / 2}$ may be replaced by 0 if $\lambda_{i}$ is too small compared to the largest singular value $\lambda_{1}$, i.e., for $i>s$ where $s$ be the number of retained singular values. Then,

$$
\Sigma^{-1 / 2}=\Lambda_{s}^{-1 / 2} U_{s}^{T} \in \mathbb{R}^{s \times h}
$$

where $\Lambda_{s}^{-1 / 2}=\operatorname{diag}\left(\lambda_{1}^{-1 / 2}, \ldots, \lambda_{s}^{-1 / 2}\right)$ and $U_{s}$ consists of the first $s$ columns of $U$.

Remark 1. The definition and computation of the considered tests from Section 3 can be extended to the case when $\Sigma$ is only positive semidefinite (Döhler and Mevel, 2013, Theorem 11), replacing $\Sigma^{-1}$ by $\Sigma^{\dagger}$, where ${ }^{\dagger}$ denotes the pseudoinverse. In this case, $\Sigma^{-1 / 2}$ is defined similarly for the non-zero singular 
values as in (14), satisfying $\Sigma^{\dagger}=\left(\Sigma^{-1 / 2}\right)^{T} \Sigma^{-1 / 2}$. This case appears especially when computing the sample covariance from measurement data, for which an efficient computation of $\Sigma^{-1 / 2}$ is detailed in (Döhler and Mevel, 2011; Döhler et al., 2014b). The numerical computations in the following sections remain valid for the rank deficient case, if the product $\Sigma^{-1 / 2} \mathcal{J}$ has full column rank, requiring in particular $s \geq l$ where $l=\operatorname{dim}(\delta)$.

\subsection{Computation of global test}

Theorem 2 (Zhang and Basseville (2003)). Let the thin $Q R$ decomposition (Golub and Van Loan, 1996)

$$
\Sigma^{-1 / 2} \mathcal{J}=Q R
$$

be given, where $Q$ is an orthonormal matrix $\left(Q^{T} Q=I\right)$ and $R$ is a square matrix. Then, the global test in (4) writes as

$$
t_{\text {global }}=\alpha^{T} \alpha, \text { where } \alpha=Q^{T} \Sigma^{-1 / 2} \zeta .
$$

Proof. Because $\Sigma$ is positive definite, $\Sigma^{-1 / 2}$ has full rank, and because $\mathcal{J}$ has full column rank, matrix $R$ is invertible. Using (13), (15) and $Q^{T} Q=I$, the global test (4) writes

$$
\begin{aligned}
t_{\text {global }} & =\zeta^{T}\left(\Sigma^{-1 / 2}\right)^{T} Q R\left(R^{T} Q^{T} Q R\right)^{-1} R^{T} Q^{T} \Sigma^{-1 / 2} \zeta \\
& =\zeta^{T}\left(\Sigma^{-1 / 2}\right)^{T} Q Q^{T} \Sigma^{-1 / 2} \zeta=\alpha^{T} \alpha .
\end{aligned}
$$

The advantage of the computation in (16) is the handling of numerical errors when $\Sigma$ or $\mathcal{J}$ are badly conditioned. For example, the direct computation of the test variable in (4) may be negative due to rounding errors, while the computation in (16) amounts to a squared Euclidean norm that is always positive. Moreover, the number of multiplications with potentially badly conditioned matrices and thus the propagation of rounding errors is reduced. The $\mathrm{QR}$ decomposition of $\Sigma^{-1 / 2} \mathcal{J}$ is used to obtain the orthonormal matrix $Q$, which is known to be numerically well behaved. The extra computational cost for obtaining $Q$ is compensated by less matrix multiplications in the test computation, in particular avoiding the computation of the Fisher information matrix and its inverse.

\subsection{Computation of isolation tests}

\subsubsection{Sensitivity test}

The computation of the sensitivity test (6) is similar to the global test. Hence, using the thin QR decomposition

$$
\Sigma^{-1 / 2} \mathcal{J}_{a}=Q_{a} R_{a}
$$

an efficient computation is given by

$$
t_{\mathrm{sens}}=\alpha_{a}^{T} \alpha_{a}, \text { where } \alpha_{a}=Q_{a}^{T} \Sigma^{-1 / 2} \zeta
$$

with the same advantages as for the global test in the previous section.

\subsubsection{Minmax test}

The computation of the minmax test (12) in Section 3.2.2 is more sophisticated. After the preliminary work in (Zhang and Basseville, 2003), we show in the following theorem that the computation simplifies significantly when using an appropriate QR decomposition, leading to a direct and efficient computation.

Theorem 3. Let the thin $Q R$ decomposition of

$$
\Sigma^{-1 / 2}\left[\begin{array}{ll}
\mathcal{J}_{b} & \mathcal{J}_{a}
\end{array}\right]=\left[\begin{array}{ll}
Q_{b} & Q_{a}
\end{array}\right]\left[\begin{array}{cc}
R_{b b} & R_{b a} \\
0 & R_{a a}
\end{array}\right]
$$

be given and partitioned accordingly. Then, the minmax test in (12) writes as

$$
t_{\mathrm{mm}}=\beta_{a}^{T} \beta_{a}, \text { where } \beta_{a}=Q_{a}^{T} \Sigma^{-1 / 2} \zeta .
$$

Proof. From the QR decomposition (19) it follows

$$
\begin{aligned}
& \Sigma^{-1 / 2} \mathcal{J}_{a}=Q_{b} R_{b a}+Q_{a} R_{a a}, \\
& \Sigma^{-1 / 2} \mathcal{J}_{b}=Q_{b} R_{b b},
\end{aligned}
$$

where $Q_{a}^{T} Q_{a}=I, Q_{b}^{T} Q_{b}=I$ and $Q_{b}^{T} Q_{a}=0$, and thus in (5)

$$
F=\left[\begin{array}{ll}
F_{a a} & F_{a b} \\
F_{b a} & F_{b b}
\end{array}\right]=\left[\begin{array}{cc}
R_{b a}^{T} R_{b a}+R_{a a}^{T} R_{a a} & R_{b a}^{T} R_{b b} \\
R_{b b}^{T} R_{b a} & R_{b b}^{T} R_{b b}
\end{array}\right] .
$$

The partial residuals in $(8 \mathrm{a})$ and $(8 \mathrm{~b})$ write as

$$
\begin{aligned}
\zeta_{a} & =R_{b a}^{T} Q_{b}^{T} \Sigma^{-1 / 2} \zeta+R_{a a}^{T} Q_{a}^{T} \Sigma^{-1 / 2} \zeta, \\
\zeta_{b} & =R_{b b}^{T} Q_{b}^{T} \Sigma^{-1 / 2} \zeta,
\end{aligned}
$$

and the robust residual in (9) yields

$$
\begin{aligned}
\zeta_{a}^{*} & =\zeta_{a}-R_{b a}^{T} R_{b b}\left(R_{b b}^{T} R_{b b}\right)^{-1} \zeta_{b} \\
& =R_{b a}^{T} Q_{b}^{T} \Sigma^{-1 / 2} \zeta+R_{a a}^{T} Q_{a}^{T} \Sigma^{-1 / 2} \zeta \\
& \quad-R_{b a}^{T} R_{b b}\left(R_{b b}^{T} R_{b b}\right)^{-1} R_{b b}^{T} Q_{b}^{T} \Sigma^{-1 / 2} \zeta \\
& =R_{a a}^{T} Q_{a}^{T} \Sigma^{-1 / 2} \zeta .
\end{aligned}
$$

Similarly, substituting the elements of $F_{a}^{*}$ in (10) with (21) yields

$$
\begin{aligned}
F_{a}^{*} & =\left(R_{b a}^{T} R_{b a}+R_{a a}^{T} R_{a a}\right)-R_{b a}^{T} R_{b b}\left(R_{b b}^{T} R_{b b}\right)^{-1} R_{b b}^{T} R_{b a} \\
& =R_{a a}^{T} R_{a a} .
\end{aligned}
$$

Finally, replacing $\zeta_{a}^{*}$ and $F_{a}^{*}$ in the minmax test (12) yields

$$
\begin{aligned}
t_{\mathrm{mm}} & =\zeta^{T}\left(\Sigma^{-1 / 2}\right)^{T} Q_{a} R_{a a}\left(R_{a a}^{T} R_{a a}\right)^{-1} R_{a a}^{T} Q_{a}^{T} \Sigma^{-1 / 2} \zeta \\
& =\zeta^{T}\left(\Sigma^{-1 / 2}\right)^{T} Q_{a} Q_{a}^{T} \Sigma^{-1 / 2} \zeta,
\end{aligned}
$$

leading to the assertion.

The computation in (20) requires less multiplications with potentially badly conditioned matrices compared to the direct computation in Section 3.2.2, avoiding thus the accumulation of rounding errors. Instead, the minmax test statistic boils down to a simpler expression using the $\mathrm{QR}$ decompositions, which is numerically well behaved.

Moreover, Theorem 3 makes the operation of the minmax test directly visible, when writing it equivalently as $t_{\mathrm{mm}}=\bar{\beta}^{T} \bar{\beta}$ 
with $\bar{\beta}=Q_{a} Q_{a}^{T} \Sigma^{-1 / 2} \zeta$. Note that $Q_{a} Q_{a}^{T}$ defines an orthogonal projection. So in fact the squared norm of a vector $\bar{\beta}$ is computed, which is the orthogonal projection of the covariancenormalized residual into the subspace of the normalized sensitivities $\Sigma^{-1 / 2} \mathcal{J}_{a}$ that are of interest and that are orthogonal to the normalized sensitivities $\Sigma^{-1 / 2} \mathcal{J}_{b}$. Like this, the projection is blind to changes $\delta_{b}$, while only possible changes $\delta_{a}$ remain in the projected vector. Note that this means in practice that the sensitivities of all parameters in $\mathcal{J}$ need to be sufficiently pairwise distinct, otherwise the effect of a tested change $\delta_{a}$ might be removed by another parameter in the projection. This also corresponds to the necessity of (numerical) invertibility of the $R$ matrix in (19) and thus of $R_{a a}$ and $R_{b b}$ in the proof of Theorem 3 , which is in theory given through the full column rank of $\mathcal{J}$ and the positive definiteness of $\Sigma$. However, if these matrices are too badly conditioned because some components of the parametrization are too close, the minmax test might not perform as expected, which is even worse in its original formulation in Section 3.2.2.

Besides, a fast iterative update of the QR decomposition for testing multiple subsets becomes possible thanks to the direct computation in the new approach, as described in the following section.

\subsubsection{Minmax tests on multiple parameter subsets}

For the purpose of fault isolation, typically different minmax tests are performed, each focusing on a different subset $\delta_{a}$ of the vector $\delta$ and requiring a QR decomposition in (19). The entire task implies a significant computational burden if the number of parameters $l$ is high. In what follows we propose a method to reduce the computational burden in such situations.

We assume $\delta_{a}$ to be one-dimensional in the following for simplicity of notation. Denote the elements of $\delta=$ $\left[\begin{array}{llll}\delta^{1} & \delta^{2} & \ldots & \delta^{l}\end{array}\right]^{T}$. Thus, when testing parameter $\delta^{i}=0$ against $\delta^{i} \neq 0$, denote $\delta_{a}$ and $\delta_{b}$ from the previous section as

$$
\begin{aligned}
\delta_{a}^{i} & =\delta^{i}, \\
\delta_{b}^{i} & =\left[\begin{array}{llllll}
\delta^{i+1} & \cdots & \delta^{l} & \delta^{1} & \cdots & \delta^{i-1}
\end{array}\right]^{T},
\end{aligned}
$$

and the corresponding sensitivity matrices as $\mathcal{J}_{a}^{i}$ and $\mathcal{J}_{b}^{i}$, respectively.

Instead of directly computing the QR decomposition in (19) for each parameter $i$, the fact that the columns of the sensitivity matrix $\mathcal{J}$ are the same all the time up to permutations will be used to reduce the cost of the $\mathrm{QR}$ decompositions. Only one thin QR decomposition is computed at the start for $i=1$. Then, an updating step is carried out iteratively for parameter $i$ to $i+1$, which is developed in the following.

Assume that the QR decomposition is available for parameter $i$ in (19) as

$$
\mathcal{K}^{i} \stackrel{\text { def }}{=} \Sigma^{-1 / 2}\left[\begin{array}{ll}
\mathcal{J}_{b}^{i} & \mathcal{J}_{a}^{i}
\end{array}\right]=Q^{i} R^{i},
$$

where $\mathcal{K}^{i} \in \mathbb{R}^{s \times l}$. At this stage, the vector $Q_{a}^{i}$ in $Q^{i}=\left[\begin{array}{ll}Q_{b}^{i} & Q_{a}^{i}\end{array}\right]$ is used for the minmax test regarding $\delta_{a}^{i}$ in Theorem 3, where the test statistic

$$
t_{\mathrm{mm}}^{i}=\left(\beta_{a}^{i}\right)^{T} \beta_{a}^{i}, \quad \text { where } \beta_{a}^{i}=\left(Q_{a}^{i}\right)^{T} \Sigma^{-1 / 2} \zeta
$$

is computed.

In the next step, the update for parameter $i+1$ will be performed to compute the respective minmax test statistic, where the decomposition

$$
\mathcal{K}^{i+1}=\Sigma^{-1 / 2}\left[\begin{array}{ll}
\mathcal{J}_{b}^{i+1} & \mathcal{J}_{a}^{i+1}
\end{array}\right]=Q^{i+1} R^{i+1} .
$$

is required. It can be obtained efficiently as follows. The matrices $\mathcal{K}^{i}$ and $\mathcal{K}^{i+1}$ are related by a permutation of one column due to the definition in (22a)-(22b): partitioning $\mathcal{K}^{i}=\left[\begin{array}{ll}k_{1} & K_{2}\end{array}\right]$ with $k_{1}$ being the first column leads to $\mathcal{K}^{i+1}=\left[\begin{array}{ll}K_{2} & k_{1}\end{array}\right]$. Partitioning $R^{i}=\left[\begin{array}{ll}r_{1} & R_{2}\end{array}\right]$ analogously, it follows $\mathcal{K}^{i}=$ $\left[\begin{array}{ll}Q^{i} r_{1} & Q^{i} R_{2}\end{array}\right]$ from (23) and thus

$$
\mathcal{K}^{i+1}=Q^{i}\left[\begin{array}{ll}
R_{2} & r_{1}
\end{array}\right] .
$$

The matrix $\left[\begin{array}{ll}R_{2} & r_{1}\end{array}\right]$ is upper Hessenberg due to the upper triangular structure of $R^{i}=\left[\begin{array}{ll}r_{1} & R_{2}\end{array}\right]$. Hence, there are Givens rotations $G_{1}, \ldots, G_{l-1}$, such that the product $G_{l-1}^{T} \cdots G_{1}^{T}\left[\begin{array}{ll}R_{2} & r_{1}\end{array}\right]$ is upper triangular (Golub and Van Loan, 1996), and the QR decomposition of the next iteration in (25) can be obtained efficiently from

$$
Q^{i+1}=Q^{i} G_{1} \cdots G_{l-1}, \quad R^{i+1}=G_{l-1}^{T} \cdots G_{1}^{T}\left[\begin{array}{ll}
R_{2} & r_{1}
\end{array}\right] .
$$

This update for $Q^{i+1}$ and $R^{i+1}$ takes only $6 s l$ flops and $3 l^{2}$ flops, respectively, compared to $4 s l^{2}-\frac{4}{3} l^{3}$ flops for the direct computation of the thin QR decomposition (Golub and Van Loan, 1996). Moreover, the explicit computation of $Q^{i+1}$ in (26) is not even required for the test computation, since it follows from (24) and (26)

$$
\begin{aligned}
\beta_{a}^{i+1} & =\left(Q_{a}^{i+1}\right)^{T} \Sigma^{-1 / 2} \zeta \\
& =G_{l-1}^{T} \cdots G_{1}^{T}\left(Q_{a}^{i}\right)^{T} \Sigma^{-1 / 2} \zeta \\
& =G_{l-1}^{T} \cdots G_{1}^{T} \beta_{a}^{i} .
\end{aligned}
$$

Thus, $\beta_{a}^{i}$ can be directly updated to $\beta_{a}^{i+1}$ based on the Givens rotations in order to compute the test $t_{\mathrm{mm}}^{i+1}=\left(\beta_{a}^{i+1}\right)^{T} \beta_{a}^{i+1}$. This update takes only $6 l$ flops, plus the $3 l^{2}$ flops for updating to $R^{i+1}$ in (26). Thus, the updating for all parameters $i=1, \ldots, l$ takes $3 l^{3}$ flops, which is of the same order as the cost of one initial QR decomposition. The QR decomposition is the dominant operation in the computation of the minmax tests, and it holds $s \geq l$. Thus, testing all parameters $i=1, \ldots, l$ with the minmax test requires altogether $O\left(s l^{2}\right)$ flops with the described iterative scheme, compared to $O\left(s l^{3}\right)$ flops based on the explicit computation of the $\mathrm{QR}$ decompositions for all parameters in Theorem 3.

\section{Fault quantification}

With the tests in the previous sections, a decision between $\delta_{a}=0$ and $\delta_{a} \neq 0$ is made for the components of the vector $\delta$ based on the sensitivity and the minmax approach. If the respective test exceeds a threshold and it is decided that the component is faulty, i.e. $\delta_{a} \neq 0$, the question for the actual value of 
$\delta_{a}$ arises. In this section, estimates of the change $\delta_{a}$ are derived based on the properties of the sensitivity and minmax tests, respectively. Special care is also taken of a numerically sensible computation of these estimates. Note that the discussion of the choice between the sensitivity and the minmax approach in Section 3.2.3 also applies to the following developments.

\subsection{Sensitivity approach}

Based on the sensitivity test from Section 3.2.1, an estimate of $\delta_{a}$ can be obtained as follows.

\section{Theorem 4. Define}

$$
\widehat{\delta}_{a}^{\text {sens }} \stackrel{\text { def }}{=}\left(\mathcal{J}_{a}^{T} \Sigma^{-1} \mathcal{J}_{a}\right)^{-1} \mathcal{J}_{a}^{T} \Sigma^{-1} \zeta
$$

Then, under the assumption $\delta_{b}=0$,

$$
\widehat{\delta}_{a}^{\text {sens }} \sim \mathcal{N}\left(\delta_{a}, F_{a a}^{-1}\right) .
$$

Proof. Since $\zeta \sim \mathcal{N}(\mathcal{J} \delta, \Sigma)$, it follows under the assumption $\delta_{b}=0$ (see Section 3.2.1) that

$$
\zeta \sim \mathcal{N}\left(\mathcal{J}_{a} \delta_{a}, \Sigma\right)
$$

It follows easily

$$
\mathcal{J}_{a}^{T} \Sigma^{-1} \zeta \sim \mathcal{N}\left(\mathcal{J}_{a}^{T} \Sigma^{-1} \mathcal{J}_{a} \delta_{a}, \mathcal{J}_{a}^{T} \Sigma^{-1} \mathcal{J}_{a}\right)
$$

and, since $F_{a a}=\mathcal{J}_{a}^{T} \Sigma^{-1} \mathcal{J}_{a}$, the assertion follows.

Since the sensitivity approach requires the assumption $\delta_{b}=$ 0 , which may be not guaranteed in reality, the potential error can be analyzed in the following corollary.

Corollary 5. For $\delta_{b}$ arbitrary, it holds

$$
\widehat{\delta}_{a}^{\text {sens }} \sim \mathcal{N}\left(\delta_{a}+F_{a a}^{-1} F_{a b} \delta_{b}, F_{a a}^{-1}\right) .
$$

Proof. Since $\zeta \sim \mathcal{N}\left(\mathcal{J}_{a} \delta_{a}+\mathcal{J}_{b} \delta_{b}, \Sigma\right)$, the assertion follows from the definition of $\widehat{\delta_{a}^{\text {sens }}}$ in (28).

Following the numerically efficient computation of the sensitivity test in Section 4.3.1, an efficient computation of $\widehat{\delta}_{a}^{\text {sens }}$ can be achieved as follows.

Corollary 6. With the $Q R$ decomposition (17), namely $\Sigma^{-1 / 2} \mathcal{J}_{a}=Q_{a} R_{a}$, it holds

$$
\widehat{\delta}_{a}^{\text {sens }}=R_{a}^{-1} Q_{a}^{T} \Sigma^{-1 / 2} \zeta,
$$

coinciding with $R_{a}^{-1} \alpha_{a}$ in the test computation in (18).

Proof. With decomposition (13), $\widehat{\delta}_{a}^{\text {sens }}$ in (28) can be written as

$$
\widehat{\delta}_{a}^{\text {sens }}=\left(\left(\Sigma^{-1 / 2} \mathcal{J}_{a}\right)^{T} \Sigma^{-1 / 2} \mathcal{J}_{a}\right)^{-1}\left(\Sigma^{-1 / 2} \mathcal{J}_{a}\right)^{T} \Sigma^{-1 / 2} \zeta .
$$

Plugging in the QR decomposition $\Sigma^{-1 / 2} \mathcal{J}_{a}=Q_{a} R_{a}$, where $Q_{a}^{T} Q_{a}=I$ and $R_{a}$ is invertible, yields

$$
\widehat{\delta}_{a}^{\text {sens }}=\left(R_{a}^{T} Q_{a}^{T} Q_{a} R_{a}\right)^{-1} R_{a}^{T} Q_{a}^{T} \Sigma^{-1 / 2} \zeta,
$$

leading to the assertion.

\subsection{Minmax approach}

Similarly to the sensitivity approach, an estimate of $\delta_{a}$ based on the minmax approach (see Section 3.2.2) can be obtained as follows.

Theorem 7. Let the variables for the minmax test be given in (8a)-(10). Define

$$
\widehat{\delta}_{a}^{\mathrm{mm}} \stackrel{\text { def }}{=}\left(F_{a}^{*}\right)^{-1} \zeta_{a}^{*}
$$

Then,

$$
\widehat{\delta}_{a}^{\mathrm{mm}} \sim \mathcal{N}\left(\delta_{a},\left(F_{a}^{*}\right)^{-1}\right)
$$

Proof. The assertion follows immediately from property (11).

Similar to the minmax test (12), the computation of $\widehat{\delta}_{a}^{\mathrm{mm}}$ in (30) is a numerical challenge since the computation of $\zeta_{a}^{*}$ and $F_{a}^{*}$ involves potentially ill-conditioned matrices that already led to numerical errors in the direct computation of the minmax test statistics. Following the numerically efficient computation of the minmax test in Section 4.3.2, an efficient computation of $\widehat{\delta}_{a}^{\mathrm{mm}}$ can be achieved as follows.

Corollary 8. With the QR decomposition (19), namely

$$
\Sigma^{-1 / 2}\left[\begin{array}{ll}
\mathcal{J}_{b} & \mathcal{J}_{a}
\end{array}\right]=\left[\begin{array}{ll}
Q_{b} & Q_{a}
\end{array}\right]\left[\begin{array}{cc}
R_{b b} & R_{b a} \\
0 & R_{a a}
\end{array}\right]
$$

it holds

$$
\widehat{\delta}_{a}^{\mathrm{mm}}=R_{a a}^{-1} Q_{a}^{T} \Sigma^{-1 / 2} \zeta,
$$

coinciding with $R_{a a}^{-1} \beta_{a}$ in the test computation in (20).

Proof. In the proof of Theorem 3 it was shown that QR decomposition (19) yields

$$
\zeta_{a}^{*}=R_{a a}^{T} Q_{a}^{T} \Sigma^{-1 / 2} \zeta, \quad F_{a}^{*}=R_{a a}^{T} R_{a a} .
$$

Substituting these results in (30) leads immediately to the assertion.

\subsection{Back to the local approach}

With the estimators developed in this section, estimates of $\delta$ are obtained corresponding to the faulty parameter components in a system parameter $\theta$ introduced in Section 2 . However, the original problem was the quantification of the actual parameter change $\theta-\theta_{0}$. Due to the local hypothesis in (2), the parameter change can be obtained from

$$
\widehat{\theta}-\theta_{0}=\widehat{\delta} / \sqrt{N}
$$

Thus, the local approach hypothesis modeling for a parameter change in $\theta$ as $\delta / \sqrt{N}$ is not only a convenient mathematical tool for establishing the CLT for the Gaussian residual, but also has a sensible and meaningful interpretation leading to an effective quantification of the actual parameter change. 


\section{Structural damage diagnosis based on the local approach}

To solve the problem of damage diagnosis in the context of vibration-based structural health monitoring (SHM) using the techniques developed in this paper, the relevant models, parameters and residuals are introduced in this section after a short overview of the SHM literature.

\subsection{Background literature}

The purpose of SHM is to determine the system health of a structure from regular (passive) observations implementing a damage identification strategy (Farrar and Worden, 2007). The particular case of vibration-based SHM has been studied from many different angles in the last decade (Carden and Fanning, 2004; Fan and Qiao, 2011). Damage detection is usually based on a reference model that is obtained from measurement data of the healthy structure, without the need of an FE model. A common strategy is to repeatedly estimate current modal parameters by means of system identification, and to compare the result to some reference modal parameters (Ramos et al., 2010; Magalhães et al., 2012). Other methods are based on model-data matching, where measurement data are directly confronted to a reference model, without resorting to repeated system identification. For instance, such methods include non-parametric change detection based on novelty detection (Worden et al., 2000; Yan et al., 2004), whiteness tests on Kalman filter innovations (Bernal, 2013) or other damage-sensitive features (Carden and Fanning, 2004).

Most methods for vibration-based damage localization infer on the parameters of a FE model of the structure, where a nominal FE model (containing stiffness, damping and mass matrices) of the structure is given in a healthy reference state. Using measured data from the damaged structure, these methods try to determine an updated model that reproduces the dynamic response from the data. Comparing the updated matrices with the original ones provides damage location and extent (Brownjohn et al., 2001). For example, FE model updating is based on changes in natural frequencies (Cawley and Adams, 1979), other modal parameters (Jaishi and Ren, 2005) and residual functions (Jaishi and Ren, 2006), or uses damage pattern functions (Teughels et al., 2002). While model updating-based approaches are in principle applicable to arbitrary structures, they are often too poorly conditioned to be successful in practice. The parameter size of FE models of real structures is usually much larger than the number of identified parameters from measurements, leading to an ill-posed problem (Friswell, 2007). Alternative methods confront measurement data to a FE model to analyse changes in the structure in a more indirect way, without updating. Empirical approaches (Fan and Qiao, 2011) and approaches with a more profound theoretical background have been developed, e.g. (Bernal, 2010) where damage is located based on structural flexibility changes, including a statistical framework for decision making on damaged and undamaged elements in (Döhler et al., 2013; Marin et al., 2015).

Compared to detection and localization, methods for damage quantification are the least developed in the literature (Fan and Qiao, 2011). Quantification may be carried out together with damage localization in the context of updating FE model parameters (Brownjohn et al., 2001; Friswell, 2007), but inherits the problem of possible ill-posedness in this case. Further methods include e.g. pattern recognition techniques based on classification principles (Abdeljaber and Avci, 2016). Alternatively, solving the localization problem first to identify the subset of changed parameters, and estimating their change in a separate second step for damage quantification, yields in general better conditioned methods, for example as is in (Bernal, 2014).

In the context of damage diagnosis based on the local approach, a subspace-based residual has been proposed in (Basseville et al., 2000; Döhler and Mevel, 2013; Döhler et al., $2014 b$ ), where it was used for damage detection. For damage localization, sensitivity tests have been introduced in (Basseville et al., 2004; Balmès et al., 2008). Within this framework, the underlying models and residuals are recalled in the following section, where they will be extended to damage localization with the appropriate minmax tests and damage quantification.

\subsection{Models, parameters and residuals}

The behavior of linear time-invariant structures subject to unknown ambient excitation can be described by the differential equation

$$
\mathcal{M} \ddot{X}(t)+C \dot{X}(t)+\mathcal{K} X(t)=v(t)
$$

where $t$ denotes continuous time; $\mathcal{M}, C, \mathcal{K} \in \mathbb{R}^{m \times m}$ are mass, damping, and stiffness matrices, respectively; the state vector $\mathcal{X}(t) \in \mathbb{R}^{m}$ is the displacement vector of the $m$ degrees of freedom of the structure; and $v(t)$ is the external unmeasured force (noise).

Observed at $r$ sensor positions (e.g. displacement, velocity or acceleration sensors) at discrete time instants $t=k \tau$ (with sampling rate $1 / \tau$ ), system (32) can also be described by a discretetime state space system model (Juang, 1994)

$$
\left\{\begin{aligned}
x_{k+1} & =A x_{k}+v_{k} \\
y_{k} & =C x_{k}+w_{k}
\end{aligned}\right.
$$

with the state vector $x_{k}=\left[\begin{array}{ll}X(k \tau)^{T} & \dot{X}(k \tau)^{T}\end{array}\right]^{T} \in \mathbb{R}^{n}$, the measured outputs $y_{k} \in \mathbb{R}^{r}$, the state transition matrix

$$
A=\exp \left(\left[\begin{array}{cc}
0 & I \\
-\mathcal{M}^{-1} \mathcal{K} & -\mathcal{M}^{-1} C
\end{array}\right] \tau\right) \in \mathbb{R}^{n \times n}
$$

and the observation matrix

$$
C=\left[L_{d}-L_{a} \mathcal{M}^{-1} \mathcal{K} \quad L_{v}-L_{a} \mathcal{M}^{-1} C\right] \in \mathbb{R}^{r \times n},
$$

where $n=2 m$ is the model order and $L_{d}, L_{v}, L_{c} \in\{0,1\}^{r \times m}$ are selection matrices indicating the positions of displacement, velocity or acceleration sensors, respectively. The state noise $v_{k}$ and output noise $w_{k}$ are unmeasured and assumed to be centered and square integrable.

Damage leads to changes in the structural properties of system (32), e.g., in mass parameters of elements in $\mathcal{M}$, or in parameters corresponding to element stiffness such as Young's modulus in $\mathcal{K}$. Hence they provoke changes in the eigenstructure of system (32), and consequently of system (33). Changes 
in the eigenstructure are directly linked to changes in the modal parameters (natural frequencies, damping ratios, mode shapes) of the structure.

Thus, two parametrizations are of interest. First, the eigenstructure parametrization $\theta^{\text {eig }}$ is the collection of eigenvalues and observed eigenvectors (mode shapes) $\left(\lambda_{i}, \varphi_{i}\right)$ of system (33) with

$$
A \phi_{i}=\lambda_{i} \phi_{i}, \varphi_{i}=C \phi_{i}, \quad i=1,2, \ldots, n
$$

It constitutes a canonical parametrization invariant to linear state transformations. An estimate of $\theta^{\text {eig }}$ can be obtained from system identification, e.g. using subspace methods that are particularly suitable for the treatment of vibration data (Van Overschee and De Moor, 1996; Peeters and De Roeck, 1999; Basseville et al., 2001; Döhler and Mevel, 2012a). Based on this parametrization, the detection of damages is possible. However, it does not contain the physical structural information that is necessary for damage localization or quantification. Second, a physical (finite element) parametrization $\theta^{\mathrm{FE}}$ is composed of damage-sensitive parameters of the elements of a structure, such as mass or stiffness parameters. Parameters in $\theta^{\mathrm{FE}}$ cannot be obtained from system identification, but are based on an FE model of the structure. Based on this parametrization, the localization and quantification of damage is possible, since each parameter in $\theta^{\mathrm{FE}}$ corresponds to properties of a limited region in the structure. It is assumed that structural damage is local and thus affects only a small part of the parameters in $\theta^{\mathrm{FE}}$.

The problems for damage detection, localization and quantification can then be formulated as follows:

- Damage detection corresponds to change detection in $\theta^{\text {eig }}$;

- Damage localization corresponds to deciding which parameters in $\theta^{\mathrm{FE}}$ are faulty (fault isolation);

- Damage quantification corresponds to estimating the changes in the faulty parameters in $\theta^{\mathrm{FE}}$.

An appropriate subspace-based residual vector has been defined in (Basseville et al., 2000) based on outputs $\mathcal{Y}_{N}=$ $\left\{y_{k}\right\}_{k=1, \ldots, N}$ of system (33) as

$$
\zeta\left(\theta_{0}, \mathcal{Y}_{N}\right) \stackrel{\text { def }}{=} \sqrt{N} \operatorname{vec}\left(S\left(\theta_{0}\right)^{T} \widehat{\mathcal{H}}_{p+1, q}\right)
$$

where $\widehat{\mathcal{H}}_{p+1, q}$ is an estimate computed on $\mathcal{Y}_{N}$ of the block Hankel matrix

$$
\mathcal{H}_{p+1, q} \stackrel{\text { def }}{=}\left[\begin{array}{cccc}
R_{1} & R_{2} & \ldots & R_{q} \\
R_{2} & R_{3} & \ldots & R_{q+1} \\
\vdots & \vdots & \ddots & \vdots \\
R_{p+1} & R_{p+2} & \ldots & R_{p+q}
\end{array}\right]
$$

containing the output correlations $R_{i}=\mathbf{E}\left(y_{k} y_{k-i}^{T}\right)$, and $S\left(\theta_{0}\right)$ is the left null space of $\mathcal{H}_{p+1, q}$ from the reference system. Assume that state and output noise of system (33) are white for simplicity, otherwise the block Hankel matrix needs to be slightly modified (Döhler and Mevel, 2012b, Remark 1). Note that the residual definition of $\zeta\left(\theta_{0}, \boldsymbol{y}_{N}\right)$ in (35) originates from the primary residual defined by $h\left(\theta_{0}, Y_{k}\right) \stackrel{\text { def }}{=} S\left(\theta_{0}\right) Y_{k}^{+}\left(Y_{k}^{-}\right)^{T}$, where

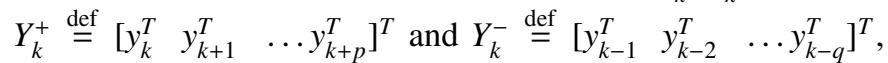
and the fact that an estimate of $\mathcal{H}_{p+1, q}$ writes as $\widehat{\mathcal{H}}_{p+1, q}=$ $\frac{1}{N} \sum_{k=1}^{N} Y_{k}^{+}\left(Y_{k}^{-}\right)^{T}$. Then, the residual vector satisfies the Central Limit Theorem (2) (Basseville et al., 2000), see Section 2. Thus, the problems of damage detection, localization and quantification are transformed to change detection and estimation on a parametrized asymptotically Gaussian residual vector, which can be solved with the methods developed in this paper. It is assumed that the data length $N$ is sufficiently large for a meaningful Gaussian approximation. Note that in either case of $\theta=\theta^{\text {eig }}$ and $\theta=\theta^{\mathrm{FE}}$, the considered changes in the parameter $\theta$ affect both the $A$ and $C$ matrices in (33), with non-additive effects on the output of the system and on the residual.

The estimation of the asymptotic residual sensitivity $\mathcal{J}$ is described in detail in (Basseville et al., 2004; Balmès et al., 2008; Döhler et al., 2014b) for both parametrizations $\theta=\theta^{\text {eig }}$ and $\theta=\theta^{\mathrm{FE}}$. In both cases, the residual is derived with respect to $\theta^{\text {eig }}$, and in the second case the derivative of $\theta^{\text {eig }}$ with respect to $\theta^{\mathrm{FE}}$ is computed in addition using FE model (32). The estimation of $\Sigma$ is described in detail in (Döhler and Mevel, 2011; Döhler et al., 2014b).

\section{Application: structural damage diagnosis}

Since damage detection in this framework has already been applied in several case studies (e.g., Siegert et al. (2010); Döhler and Hille (2014); Döhler et al. (2014a)), we focus on damage localization and quantification for application in this section. Using an FE parametrization $\theta=\theta^{\mathrm{FE}}=\left[\begin{array}{lll}\theta^{1} & \ldots & \theta^{l}\end{array}\right]$ of the investigated structure, damage localization is carried out using the sensitivity and the minmax tests for each of the parameters $\theta^{i}, i=1, \ldots, l$ with the numerically efficient computations from Sections 4.3.1 and 4.3.2. If the respective test value for a parameter exceeds a threshold, it is regarded as faulty and damage is located in the corresponding structural element. Finally, the quantification of the damage extent can be performed on the damaged elements with the respective estimators derived in Section 5.

The damage localization and quantification techniques have been applied to two simulated structures. The first example is a simple mass-spring chain, the second a truss structure. In both cases, output-only datasets with displacement samples were generated at the sensor coordinates in reference and damaged states from white noise excitation. Measurement noise was added with a magnitude of $5 \%$ of the standard deviation of each generated output signal. The damping was defined such that all modes have a damping ratio of $2 \%$. All parameters of the tests $(S, \mathcal{J}, \Sigma)$ were estimated based on the dataset in the reference state and the information from the respective FE model. In each damage quantification example, 100 realizations of the simulated time series were used. The quantification results are shown for several damage cases and extents, each as the mean from the 100 estimates together with their standard deviation. 


\subsection{Mass-spring chain}

A mass-spring chain with eight elements is considered (Fig. 1) with masses $m_{1}=m_{3}=m_{5}=m_{7}=1, m_{2}=m_{4}=$ $m_{6}=m_{8}=2$ and stiffnesses $k_{1}=k_{3}=k_{5}=k_{7}=200, k_{2}=k_{4}=$ $k_{6}=k_{8}=100$. Output time series of length $N=100,000$ are simulated at time step $\tau=0.05 \mathrm{~s}$ at four elements. The model order is $n=16$, and $p+1=q=6$ in (35). $\mathcal{J}$ is computed on all eight modes of the system. We consider three damage cases, where damage is simulated by a stiffness reduction in one or two springs.

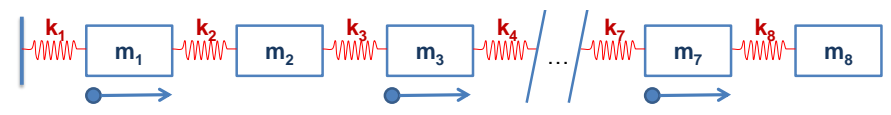

Figure 1: Mass-spring chain with four sensors.

\subsubsection{Damage localization}

Damage in element 4, Fig. 2. While the minmax tests behave perfectly, the sensitivity test for element 3 also reacts due to the violation of $\delta_{b}=0$. The strong reaction only for element 3 among the undamaged elements becomes clear when evaluating the non-centrality parameter of the test in (7), in particular the value of $F_{b a} F_{a a}^{-1} F_{a b}$ where ' $a$ ' corresponds to any undamaged element and ' $b$ ' corresponds to the damaged element 4 : this value is at 22.3 for element 3 while being lower than 0.4 for all other undamaged elements.

Damage in elements 2 and 4, Fig. 3. The minmax tests behave perfectly again, while the test value for the damage in spring 2 is of similar magnitude as the false-positives for springs 1 and 3 . This is analogous to the previous case, where it was explained why the test for element 3 reacts when element 4 is damaged (see Fig. 2). The minmax test is robust to changes in the nontested parameter changes $\delta_{b}$ by design.

Damage in elements 3 and 4, Fig. 4. The sensitivity and minmax tests behave very well in this case. Note that the test values for element 3 are in both tests larger than for element 4 , while element 4 is more damaged. Indeed, the test values serve only for the decision if the respective element is damaged or not by comparing them to a threshold. They are not directly linked to the damage extent, which is estimated separately in the following as shown in Section 5.

\subsubsection{Damage quantification}

Damage in element 4, Fig. 5 (top). The damage extents are well estimated for both the sensitivity and the minmax approaches, only large extents are slightly overestimated.

Damage in elements 2 and 4, Fig. 5 (left). The stiffness reduction in element 2 is half as large as in element 4 for each damage. Results from the minmax approach are satisfying, where again only large damage extents are slightly overestimated. The damage quantification from the sensitivity approach is biased (here underestimated), since the assumption $\delta_{b}=0$ is violated.
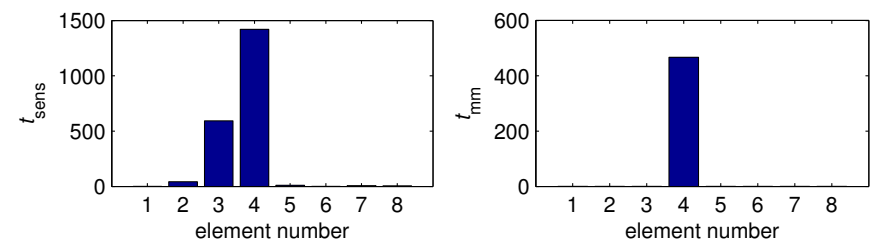

Figure 2: Sensitivity tests (left) and minmax tests (right) for $10 \%$ damage in element 4.
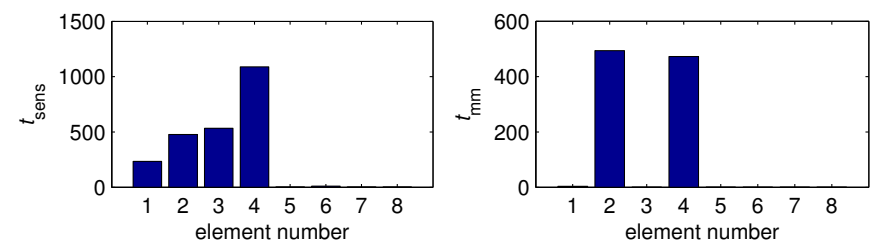

Figure 3: Sensitivity (left) and minmax tests (right) for 5\% damage in element 2 and $10 \%$ damage in element 4.
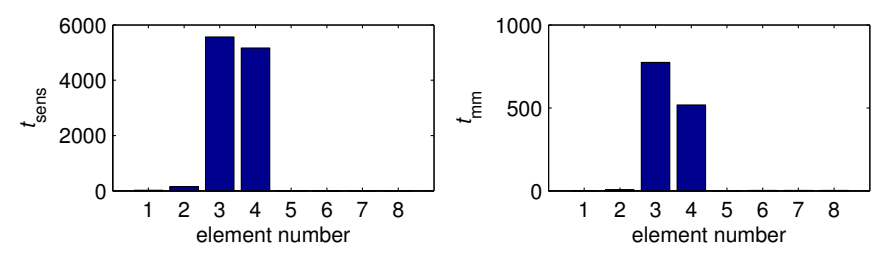

Figure 4: Sensitivity (left) and minmax tests (right) for 5\% damage in element 3 and $10 \%$ damage in element 4.

As shown in Corollary 5, the theoretic bias for $\delta_{a}$ is $F_{a a}^{-1} F_{a b} \delta_{b}$ in (29), and $F_{a a}>0$ by definition. Indeed, $\widehat{F}_{a b}=-0.4<0$ between the parameters corresponding to damaged elements 2 and 4 in this case, which explains the underestimation.

Damage in elements 3 and 4, Fig. 5 (right). The results for the minmax approach are similar as in the previous two-damage case, whereas the damage extent is strongly overestimated now when using the sensitivity approach. This is in line with $\widehat{F}_{a b}=$ $2.09>0$ for the parameters corresponding to elements 3 and 4 in this case.

\subsubsection{Numerical robustness}

The effectiveness of the proposed numerical computation of the test variables and fault estimation from Sections 4.2, 4.3.1, 4.3.2 and 5 is illustrated in this section.

The configuration of the mass-spring chain in the previous sections led to a well-conditioned example due to a small number of elements, known model order, equal noise properties on the outputs etc. In this case, there is no significant difference between the direct and the proposed numerical computations. However, the situation is different for ill-conditioned examples as shown in the following.

Such an example is defined based on the previous massspring chain with few modifications. We assume different measurement noise properties for the four outputs, namely $100 \%$ 

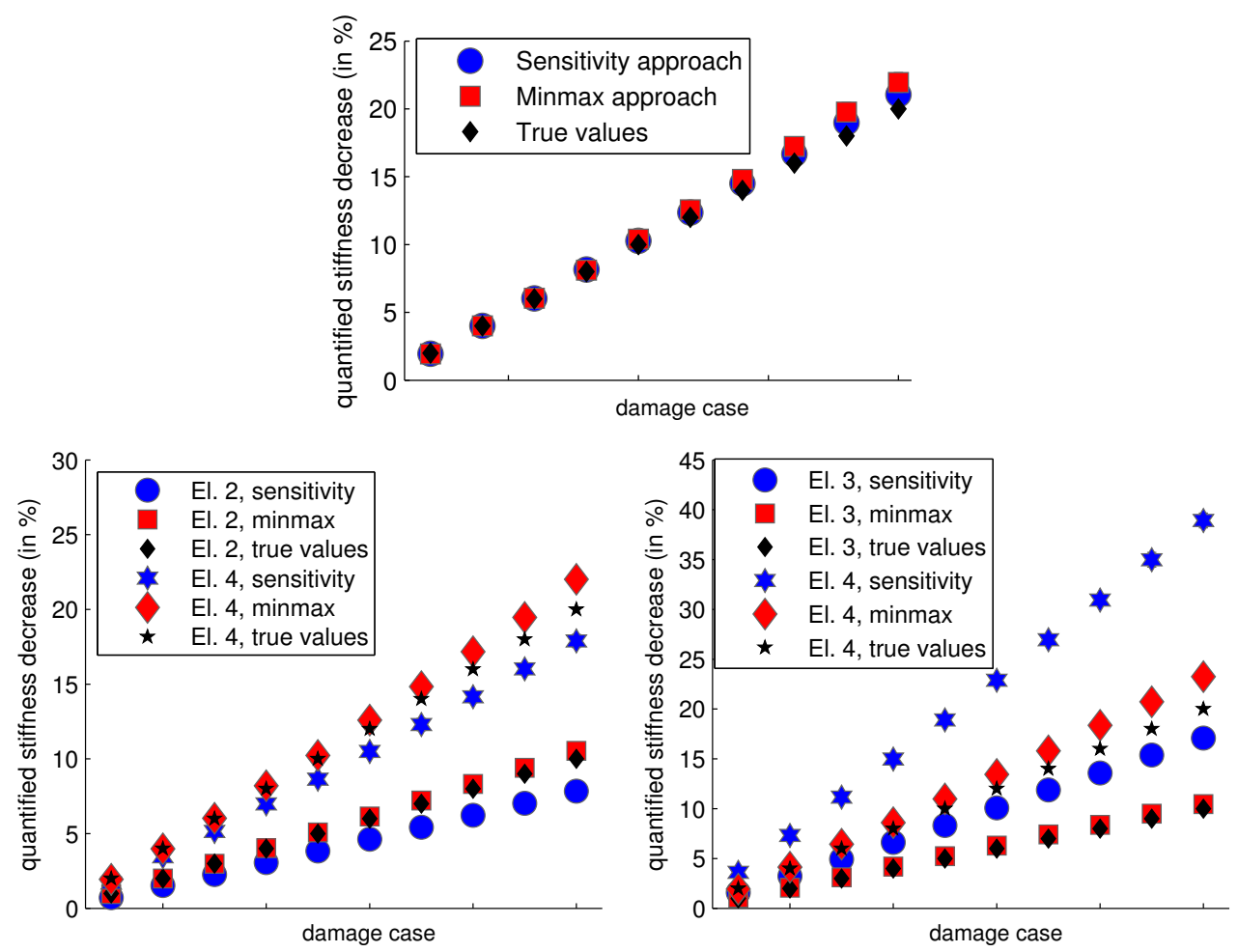

Figure 5: Quantification of different damage extents in elements 4 only (top), 2 and 4 (left), 3 and 4 (right).

measurement noise for the first sensor and $1 \%$ measurement noise for each of the three other sensors with respect to the standard deviation of the signals. Such a situation could correspond e.g. to a malfunctioning noisy sensor. Furthermore, we assume $p+1=q=9$ and the model order $n=16$ in the residual definition. The sensitivity and covariance matrices $\mathcal{J}$ and $\Sigma$ are estimated from a large data sample of length $N=1,000,000$. Their condition numbers are 336 and $2.4 \cdot 10^{11}$, respectively.

In all following test cases, 10,000 realizations of time series with length $N=100,000$ are obtained from random white noise excitation and the corresponding test statistic or estimate is computed for each realization. In each case, the direct computation and the proposed numerical computation are carried out. From these values, the empirical probability density is obtained in a histogram and in a kernel pdf estimate, which is compared to the theoretical pdf of the underlying distribution.

Global test and sensitivity test. Since both the global and the sensitivity tests have a similar derivation, only the global test is considered for an illustration in this section. Consider two cases, first the system in the reference condition, and second a $1 \%$ stiffness loss in element 3 . In both cases, the test statistic $t_{\text {global }}$ is $\chi^{2}$ distributed with 8 degrees of freedom, and in the second case the non-centrality parameter is 60.9.

In both cases, there is a significant difference between the theoretical density functions and the results of the direct computation of the test statistic (Fig. 6 and 7, left), where even negative values appear. In contrast, there is a much better agreement between the results of the proposed numerical computation and the theoretic density function (Fig. 6 and 7, right), indicating the good numeric behavior of the algorithm. The small discrepancies between the numerical results and the theoretic densities may be due to the fact that the residual distribution is not perfectly Gaussian, but only approximated by a Gaussian through the CLT.

To decide between reference and faulty states, a threshold can be set up based on the theoretical $\chi^{2}(8)$ distribution in the reference system for a given probability of false alarms. For example, this threshold is set at 20.09 when allowing a $1 \%$ false alarm rate. Then, the empirical false alarm rate corresponding to the results in Fig. 6 is $18.4 \%$ for the direct computation, which would impair strongly the applicability of the test in practice, and $1.9 \%$ for the proposed numerical computation. The probability of detection corresponding to the results in Fig. 6 is then only $84.7 \%$ for the direct computation, but $100 \%$ for the proposed numerical computation. Note that in practice the threshold can also be set from the pdf estimate instead of the theoretical distribution of the reference state. In this case, it would be set at 91.3 for a $1 \%$ false alarm rate with the direct computation, leading to a probability of detection of only $21.9 \%$. In the proposed numerical computation, the empirical threshold would be set at 22.1 , still leading to $100 \%$ probability of detection. Hence, the proposed numerical computation clearly outperforms the direct computation.

Minmax test. Consider again 1\% stiffness loss in element 3, and consider two elements being tested for damage. In the first case, element 1 is tested $\left(\delta_{a}=0, \delta_{b} \neq 0\right)$ and in the second case 

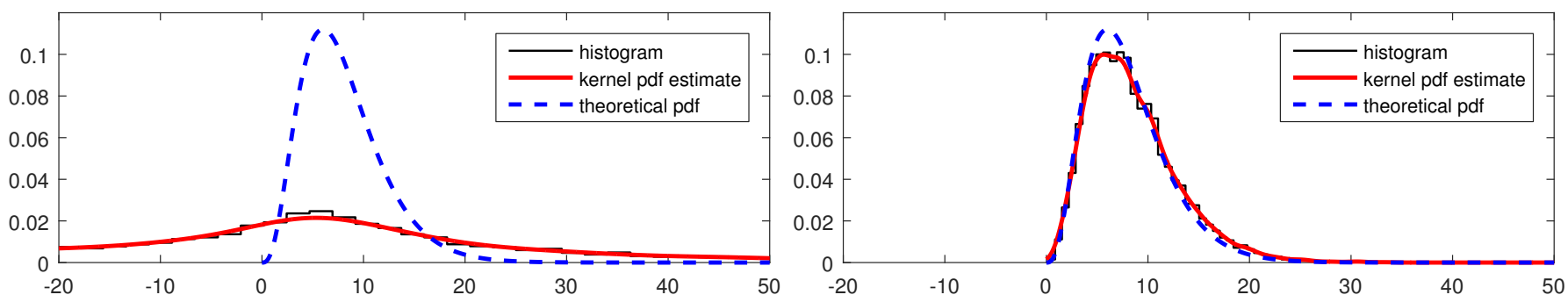

Figure 6: Global tests from reference system with central distribution $\chi^{2}(8)$. Direct (left) and proposed numerical (right) computation.
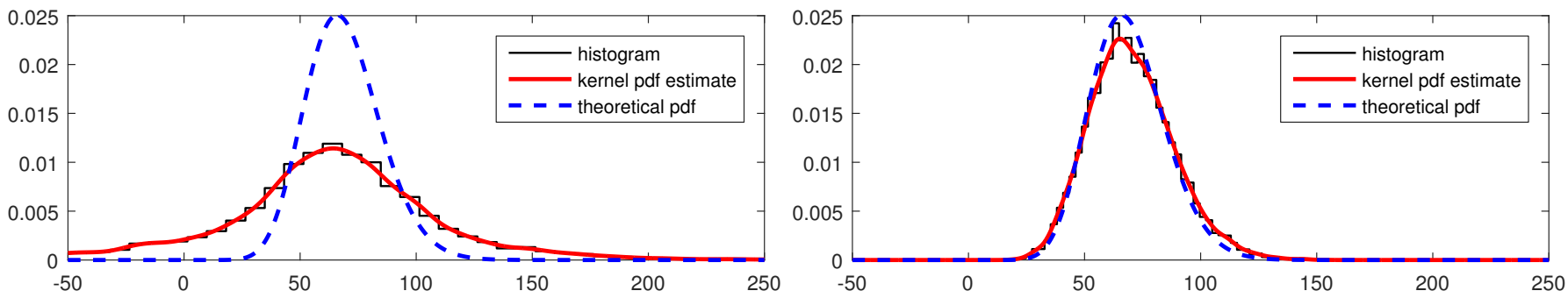

Figure 7: Global tests from damaged system with non-central distribution $\chi^{2}(8,60.9)$. Direct (left) and proposed numerical (right) computation.
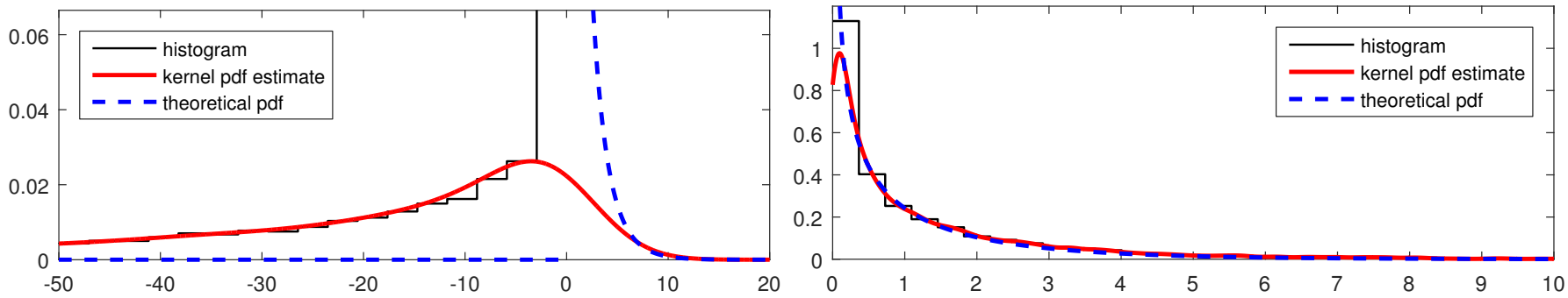

Figure 8: Minmax tests for element 1 in reference state (central distribution $\chi^{2}(1)$ ), while element 3 is damaged. Direct (left) and proposed numerical (right) computation.
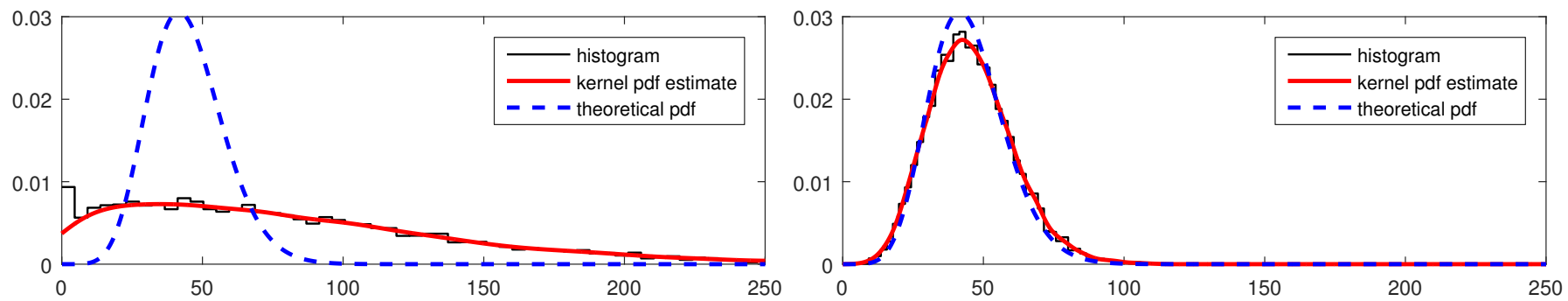

Figure 9: Minmax tests for damaged element 3 (non-central distribution $\chi^{2}(1,43.6)$ ). Direct (left) and proposed numerical (right) computation.

element 3 is tested $\left(\delta_{a}=-0.02 \sqrt{N}, \delta_{b}=0\right)$. In both cases, the test statistic $t_{\mathrm{mm}}$ is $\chi^{2}$ distributed with 1 degree of freedom, and in the second case the non-centrality parameter is 43.6 .

As in the previous example, there is a significant difference between the theoretical density function and the results from the direct computation (Fig. 8 and 9, left). In particular, all test values in Fig. 8, left, are negative. The agreement between the results of the proposed numerical computation and the theoretical density function for this minmax test is much better (Fig. 8 and 9, right), indicating the good numeric behavior of the algo- rithm. The necessity of an efficient numerical computation of the minmax test statistic is evident.

Furthermore, the probability of detection is higher with the proposed numerical computation, as in the previous example. For a threshold based on the $\chi^{2}(1)$ distribution at a $1 \%$ false alarm rate, set at 6.63 , the probability of detection of the damage in element 3 is $94.6 \%$ with the direct computation, while being at $100 \%$ with the proposed numerical computation. The test results with the direct computation for element 1 cannot be evaluated since they are negative. 

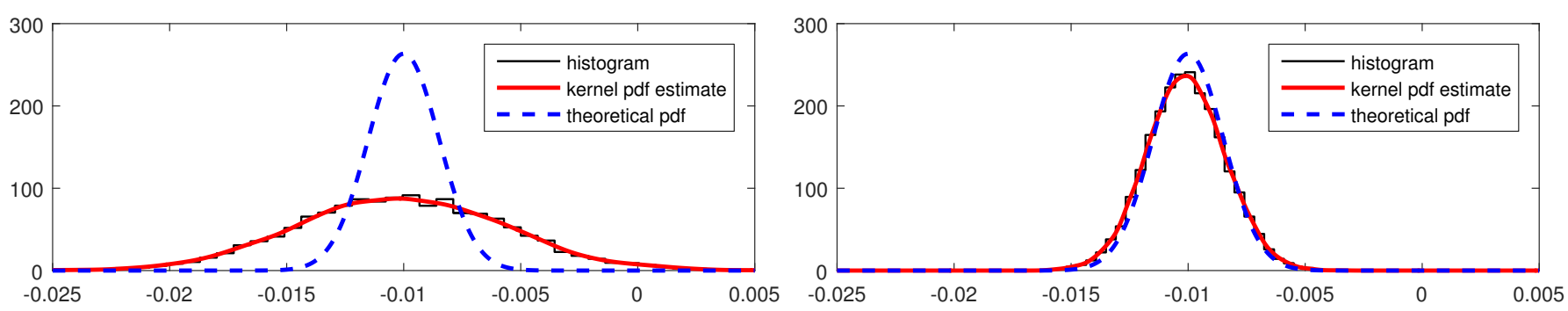

Figure 10: Direct (left) and proposed numerical (right) computation of the fault extent $\delta_{a}^{\mathrm{mm}} / \sqrt{N}$ with normal distribution $\mathcal{N}(-0.02,0.23 / N)$ for element 3 .

Fault quantification. Based on the previous minmax test case, the fault extent in element 3 is estimated, using either the direct computation in (30) or the numerically efficient computation in (31). The estimate is normal distributed as shown in Theorem 7. After normalizing by $\sqrt{N}$, both the direct and numerically efficient computations led to the approximately same mean value -0.0101 of $\widehat{\delta}_{a}^{\mathrm{mm}} / \sqrt{N}$ from the 10,000 realizations. However, the empirical distribution resulting from the direct computation deviates significantly from the theoretical one, having a larger variance, while the the results from the numerically efficient computation are much closer to the theoretical one, as shown in Fig. 10.

\subsection{Truss}

A truss structure with 25 elements of equal stiffness properties, having six sensors, has been considered as a more complex example (Fig. 11). Output time series of length $N=50,000$ are simulated at time step $\tau=0.05 \mathrm{~s}$. At this sampling frequency, we are in the more realistic case of modal truncation, since only ten modes are present in the data that can be taken into account for the computation of $\mathcal{J}$, compared to altogether 25 modes of the analytical model. In general, only a small number of modes can be obtained from measurements of system (33) compared to the number of modes present in the FE model (32). Furthermore, $S$ is estimated on the simulated data in this example and not on the analytical modes of the model. Damage is simulated by decreasing the element stiffness.

Two scenarios are considered, one with damage in element 16 , and another one with damage in both elements 16 and 23, where the stiffness reduction in element 16 is half as large as in element 23 for each considered damage extent. We show only quantification results for these cases and skip localization results for brevity. Still, damage was localized correctly in both cases with the sensitivity and the minmax tests, where the test values for the undamaged elements are much lower in the minmax tests, as expected.

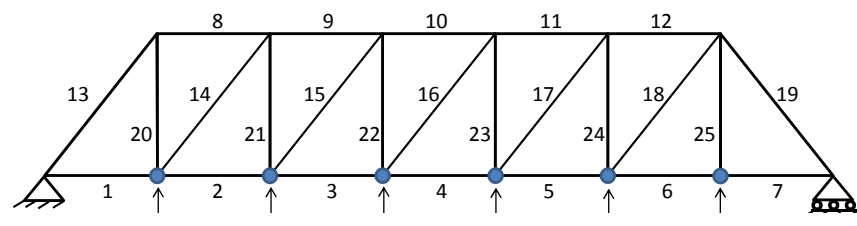

Figure 11: Truss structure with six sensors
Damage in element 16, Fig. 12 (left). The estimates from both the sensitivity and the minmax approach are close to the true values. The estimation errors are however larger for large damage extents, maybe due to the nature of the local approach: the relation between $\zeta$ and $\delta$ is non-linear and the first-order approximation involving $\mathcal{J}$ (computed at $\delta=0$ ) may thus be less accurate for large changes $\delta$.

Damage in elements 16 and 23, Fig. 12 (right). The minmax approach yields quite accurate estimates, while the sensitivity approach overestimates the damage extent significantly, similar to the example in Fig. 5 (right). This overestimation can be explained analogously by $\widehat{F}_{a b}=0.2>0$ between damaged elements 16 and 23.

While this example does not satisfy the theoretical assumptions perfectly anymore (modal truncation), it still gives reasonable results. This shows that the developed damage quantification approach is promising, and further investigation is required for application on real structures. Furthermore, the problem of robustness to environmental changes is important in real SHM applications, which can be accommodated by using modified residual functions or parameter sets in the presented methods, e.g. for temperature changes as in (Balmès et al., 2009) or for changes in the ambient excitation as in (Döhler and Mevel, 2013).

\section{Discussion and conclusions}

In this paper, FDI and fault quantification have been investigated in a common framework based the local approach to change detection and on statistical tests. It has been shown that isolation of faulty parameters is effectively possible using minmax tests. Since the direct computation of the respective test statistics may lead to large numerical errors when the residual sensitivity or covariance are ill-conditioned, we have developed robust numerical schemes for their computation based on the pertinent use of QR decompositions. Furthermore, it has been shown that the local approach is not only a convenient mathematical tool for residual evaluation in a Gaussian framework, but also provides an effective solution for the quantification of the parameter change. Based on sensitivity and minmax tests, estimators for the parameter change have been derived together with QR based robust numerical schemes for their computation.

While the sensitivity test has a simpler computation than the minmax test, it uses stronger assumptions on the parameter 

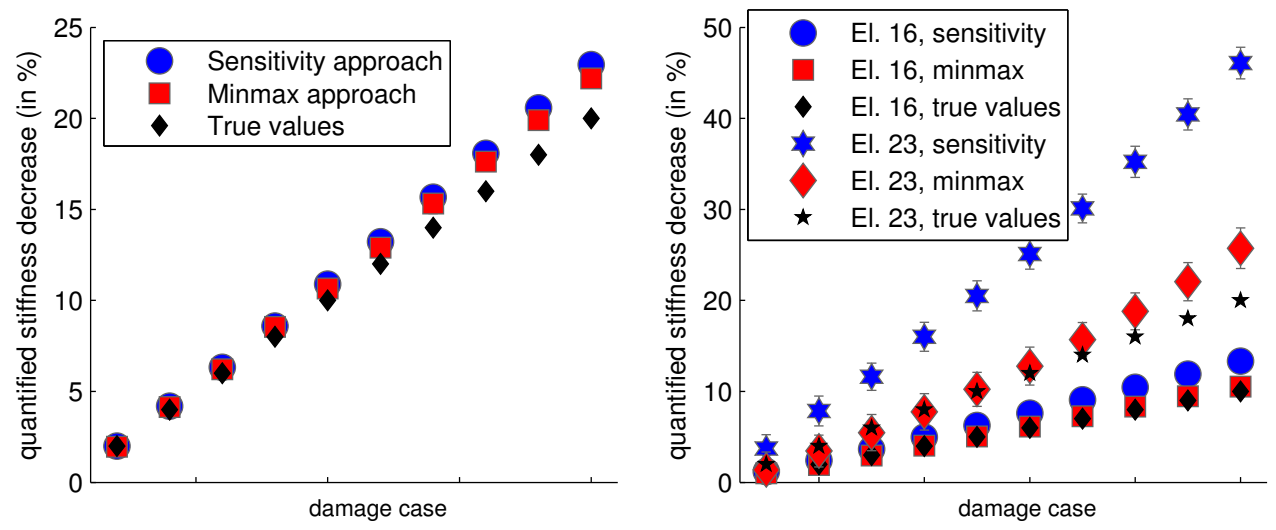

Figure 12: Quantification of different damage extents in element 16 (left) and both elements 16 and 23 (right).

change and should only be used for the special case of testing the faulty parameter for the case of single faults, or when the normalized parameter sensitivities are orthogonal. Considering that these conditions are usually unknown beforehand or can hardly be guaranteed, the minmax approach should be chosen by default.

Two simulation examples of vibration-based structural damage localization and quantification have been presented. It has been confirmed that the local approach assumption is relevant if parameter changes are small. With simultaneous parameter changes, i.e. more than one damaged elements, the minmax test proved to be more effective than the sensitivity test for both damage localization and quantification. Further work should address the problem that FE model dimensions largely exceed the modal parameter dimension, which is related to the number of sensors and identified modes. Compressed sensing seems a promising tool in this perspective, whereas its application to structural damage diagnosis problems, or more generally to FDI in complex dynamic systems, remains a challenging research topic.

\section{References}

Abdeljaber, O., Avci, O., 2016. Nonparametric structural damage detection algorithm for ambient vibration response: Utilizing artificial neural networks and self-organizing maps. Journal of Architectural Engineering 22 (2), 04016004

Balmès, E., Basseville, M., Mevel, L., Nasser, H., 2009. Handling the temperature effect in vibration-based monitoring of civil structures: a combined subspace-based and nuisance rejection approach. Control Engineering Practice 17 (1), 80-87.

Balmès, E., Basseville, M., Mevel, L., Nasser, H., Zhou, W., 2008. Statistical model-based damage localization: a combined subspace-based and substructuring approach. Structural Control and Health Monitoring 15 (6), 857 875

Basseville, M., 1997. Information criteria for residual generation and fault detection and isolation. Automatica 33 (5), 783-803.

Basseville, M., Abdelghani, M., Benveniste, A., 2000. Subspace-based fault detection algorithms for vibration monitoring. Automatica 36 (1), 101-109.

Basseville, M., Benveniste, A., Goursat, M., Hermans, L., Mevel, L., Van der Auweraer, H., 2001. Output-only subspace-based structural identification: from theory to industrial testing practice. Journal of Dynamic Systems, Measurement, and Control 123 (4), 668-676.
Basseville, M., Mevel, L., Goursat, M., 2004. Statistical model-based damage detection and localization: subspace-based residuals and damage-to-noise sensitivity ratios. Journal of Sound and Vibration 275 (3), 769-794.

Basseville, M., Nikiforov, I., 1993. Detection of Abrupt Changes - Theory and Applications. Prentice Hall, Englewood Cliffs, NJ.

Benveniste, A., Basseville, M., Moustakides, G., 1987. The asymptotic local approach to change detection and model validation. IEEE Transactions on Automatic Control 32 (7), 583-592.

Bernal, D., 2010. Load vectors for damage location in systems identified from operational loads. Journal of Engineering Mechanics 136 (1), 31-39.

Bernal, D., 2013. Kalman filter damage detection in the presence of changing process and measurement noise. Mechanical Systems and Signal Processing 39 (1-2), 361-371.

Bernal, D., 2014. Damage localization and quantification from the image of changes in flexibility. Journal of Engineering Mechanics 140 (2), 279-286.

Besançon, G., 2003. High gain observation with disturbance attenuation and application to robust fault detection. Automatica 39 (6), 1095-1102.

Blanke, M., Kinnaert, M., Lunze, J., Staroswiecki, J., 2015. Diagnosis and Fault-Tolerant Control, 3rd Edition. Springer.

Brownjohn, J., 2007. Structural health monitoring of civil infrastructure. Philosophical Transactions of the Royal Society of London A: Mathematical, Physical and Engineering Sciences 365 (1851), 589-622.

Brownjohn, J. M. W., Xia, P.-Q., Hao, H., Xia, Y., 2001. Civil structure condition assessment by FE model updating: methodology and case studies. Finite Elements in Analysis and Design 37 (10), 761-775.

Carden, E., Fanning, P., 2004. Vibration based condition monitoring: a review. Structural Health Monitoring 3 (4), 355-377.

Cawley, P., Adams, R., 1979. The location of defects in structures from measurements of natural frequencies. Journal of Strain Analysis for Engineering Design 14 (2), 49-57.

Chen, J., Patton, R. J., 1999. Robust Model-Based Fault Diagnosis for Dynamic Systems. Kluwer, Boston, USA.

Chen, R., Speyer, J., 2000. A generalized least-squares fault detection filter. Int. Jal Adaptive Control Signal Processing 14 (7), 747-757.

Ding, S. X., 2008. Model-Based Fault Diagnosis Techniques - Design Schemes Algorithms and tools. Springer-Verlag.

Döhler, M., Hille, F., 2014. Subspace-based damage detection on steel frame structure under changing excitation. In: Proc. 32nd International Modal Analysis Conference. Orlando, FL, USA.

Döhler, M., Hille, F., Mevel, L., Rücker, W., 2014a. Structural health monitoring with statistical methods during progressive damage test of S101 Bridge. Engineering Structures 69, 183-193.

Döhler, M., Marin, L., Bernal, D., Mevel, L., 2013. Statistical decision making for damage localization with stochastic load vectors. Mechanical Systems and Signal Processing 39 (1-2), 426-440.

Döhler, M., Mevel, L., 2011. Robust subspace based fault detection. In: Proc. 18th IFAC World Congress. Milan, Italy.

Döhler, M., Mevel, L., 2012a. Fast multi-order computation of system matrices in subspace-based system identification. Control Engineering Practice 20 (9), 882-894. 
Döhler, M., Mevel, L., 2012b. Modular subspace-based system identification from multi-setup measurements. IEEE Transactions on Automatic Control 57 (11), 2951-2956.

Döhler, M., Mevel, L., 2013. Subspace-based fault detection robust to changes in the noise covariances. Automatica 49 (9), 2734-2743.

Döhler, M., Mevel, L., Hille, F., 2014b. Subspace-based damage detection under changes in the ambient excitation statistics. Mechanical Systems and Signal Processing 45 (1), 207-224.

Dong, J., Verhaegen, M., 2009. Subspace based fault detection and identification for LTI systems. In: Proc. 8th IFAC Symposium on Fault Detection, Diagnosis and Safety of Technical Processes (SAFEPROCESS). Barcelona, Spain, pp. 330-335.

Dong, J., Verhaegen, M., Gustafsson, F., 2012. Robust fault detection with statistical uncertainty in identified parameters. IEEE Transactions on Signal Processing 60 (10), 5064-5076.

Edelmayer, A., Bokor, J., Szigeti, F., Keviczky, L., 1997. Robust detection filter design in the presence of time-varying system perturbations. Automatica 33, 471-475.

Escobet, T., Puig, V., Quevedo, J., Garcia, D., 2014. A methodology for incipient fault detection. In: IEEE Conference on Control Applications (CCA). IEEE, pp. 104-109.

Fan, W., Qiao, P., 2011. Vibration-based damage identification methods: a review and comparative study. Structural Health Monitoring 10 (1), 83-111.

Farrar, C., Worden, K., 2007. An introduction to structural health monitoring. Philosophical Transactions of the Royal Society A: Mathematical, Physical and Engineering Sciences 365 (1851), 303-315.

Friswell, M., 2007. Damage identification using inverse methods. Philosophical Transactions of the Royal Society A: Mathematical, Physical and Engineering Sciences 365 (1851), 393-410.

Gertler, J., 1997. Fault detection and isolation using parity relations. Control Engineering Practice 5 (5), 653-661.

Gertler, J. J., 1998. Fault Detection and Diagnosis in Engineering Systems. Marcel Dekker, New York, USA.

Golub, G., Van Loan, C., 1996. Matrix computations, 3rd Edition. Johns Hopkins University Press.

Hammouri, H., Kinnaert, M., El Yaagoubi, E. H., 1998. Observer-based approach to fault detection and isolation for nonlinear systems. IEEE Trans. on Automatic Control 44 (10), 1879-1884.

Hammouri, H., Tmar, Z., 2010. Unknown input observer for state affine systems: A necessary and sufficient condition. Automatica 46 (2), 271-278.

Harmouche, J., Delpha, C., Diallo, D., 2014. Incipient fault detection and diagnosis based on Kullback-Leibler divergence using principal component analysis: Part i. Signal Processing 94, 278-287.

Henry, D., Cieslak, J., Zolghadri, A., Efimov, D., 2014. A non-conservative $H_{-} / H_{\infty}$ solution for early and robust fault diagnosis in aircraft control surface servo-loops. Control Engineering Practice 31, 183-199.

Hwang, I., Kim, S., Kim, Y., Seah, C. E., 2010. A survey of fault detection, isolation, and reconfiguration methods. IEEE Transactions on Control Systems Technology 18 (3), 636-653.

Isermann, R., 2005. Model-based fault-detection and diagnosis-status and applications. Annual Reviews in control 29 (1), 71-85.

Isermann, R., 2006. Fault-Diagnosis System. Springer, Berlin.

Jaishi, B., Ren, W., 2005. Structural finite element model updating using ambient vibration test results. Journal of Structural Engineering 131 (4), 617628

Jaishi, B., Ren, W., 2006. Damage detection by finite element model updating using modal flexibility residual. Journal of Sound and Vibration 290 (1-2), 369-387.

Juang, J.-N., 1994. Applied system identification. Prentice Hall, Englewood Cliffs, NJ, USA.

Korbicz, J., 2004. Fault Diagnosis: Models, Artificial Intelligence, Applications. Springer-Verlag GmbH.

Le Cam, L., 1956. On the asymptotic theory of estimation and testing hypotheses. In: Proc. 3rd Berkeley Symposium on Mathematical Statistics and Probability. University of California, pp. 129-156.

Le Cam, L., 1986. Asymptotic Methods in Statistical Decision Theory. Series in Statistics. Springer, NY.

Magalhães, F., Cunha, A., Caetano, E., 2012. Vibration based structural health monitoring of an arch bridge: From automated OMA to damage detection. Mechanical Systems and Signal Processing 28, 212-228.

Marin, L., Döhler, M., Bernal, D., Mevel, L., 2015. Robust statistical dam- age localization with stochastic load vectors. Structural Control and Health Monitoring 22 (3), 557-573.

Massoumnia, M.-A., 1986. A geometric approach to the synthesis of failure detection filters. IEEE Trans. Automatic Control 31 (9), 839-846.

Medvedev, A., 1995. Fault detection and isolation by a continuous parity space method. Automatica 31 (7), 1039-1044.

Peeters, B., De Roeck, G., 1999. Reference-based stochastic subspace identification for output-only modal analysis. Mechanical Systems and Signal Processing 13 (6), 855-878.

Puig, V., 2010. Fault diagnosis and fault tolerant control using set-membership approaches: Application to real case studies. International Journal of Applied Mathematics and Computer Science 20 (4), 619-635.

Raka, S., Combastel, C., 2013. Fault detection based on robust adaptive thresholds: A dynamic interval approach. Annual Reviews in Control 37, 119-128.

Ramos, L., Marques, L., Lourenço, P., De Roeck, G., Campos-Costa, A., Roque, J., 2010. Monitoring historical masonry structures with operational modal analysis: Two case studies. Mechanical Systems and Signal Processing 24 (5), 1291-1305.

Siegert, D., Döhler, M., Ben Mekki, O., Mevel, L., Goursat, M., Toutlemonde, F., 2010. Vibration monitoring of a small span composite bridge. In: Proc. 28th International Modal Analysis Conference. Jacksonville, FL, USA.

Simani, S., Fantuzzi, C., Patton, R., Patton, R., 2003. Model-based Fault Diagnosis in Dynamic Systems Using Identification Techniques. Springer.

Tan, C., Edwards, C., 2002. Sliding mode observers for detection and reconstruction of sensor faults. Automatica 38 (10), 1815-1821.

Teughels, A., Maeck, J., De Roeck, G., 2002. Damage assessment by FE model updating using damage functions. Computers \& Structures 80 (25), 1869 1879.

Van Overschee, P., De Moor, B., 1996. Subspace Identification for Linear Systems: Theory, Implementation, Applications. Kluwer.

Worden, K., Manson, G., Fieller, N., 2000. Damage detection using outlier analysis. Journal of Sound and Vibration 229 (3), 647-667.

$\mathrm{Xu}$, A., Zhang, Q., 2004. Nonlinear system fault diagnosis based on adaptive estimation. Automatica 40 (7), 1181-1193.

Yan, A., De Boe, P., Golinval, J., 2004. Structural damage diagnosis by Kalman model based on stochastic subspace identification. Structural Health Monitoring 3 (2), 103-119.

Zhang, Q., Basseville, M., 2003. Advanced numerical computation of $\chi^{2}$-tests for fault detection and isolation. In: 5th IFAC Symposium on Fault Detection, Supervision and Safety for Technical Processes (SAFEPROCESS). Washington, USA, pp. 211-216.

Zhong, M., Zhou, D., Ding, S., 2010. On designing $\mathcal{H}_{\infty}$ fault detection filter for linear discrete time-varying systems. IEEE Trans. Automatic Control 55 (7), 1689-1695.

Zouari, R., Mevel, L., Basseville, M., 2009. Subspace-based damping monitoring. In: Proc. 15th IFAC Symposium on System Identification (SYSID). Saint-Malo, France, pp. 850-855.

Michael Döhler is Chargé de Recherche at Inria (French National Institute for Research in Computer Science and Automatic Control) in Rennes, France. He received his Diploma in Technomathematics from Chemnitz University of Technology, Germany, in 2008, and his Ph.D. in Applied Mathematics from University of Rennes 1 in 2011. After postdoctoral stays at Northeastern University, Boston, USA (2012) and BAM Federal Institute of Material Research and Testing, Berlin, Germany (2013) he joint Inria as a research scientist. His research interests are in statistical methods for structural vibration analysis, in particular for system identification, uncertainty quantification and fault diagnosis.

Laurent Mevel is Directeur de Recherche at Inria in Rennes, France. He graduated from University of Rennes 1 in 1997, where he received a Ph.D. in Applied Mathematics in the field of statistical inference for hidden Markov models. Now he is working on identification and detection of partially hidden 
stochastic systems. His interests include: structural health monitoring and detection for vibration mechanics, modeling and rejection of environmental effects, model validation, uncertainty estimation and model reduction of large systems.

Qinghua Zhang received the B.S. degree from the University of Science and Technology of China in 1986, the D.E.A. (Diplôme d'Etude Approfondie) from Université de Lille 1, France, in 1988, the Ph.D. and the H.D.R. (Habilitation Diriger des Recherches) both from Université de Rennes 1, France, in 1991 and 1999, respectively. During 1992, he was a postdoctorant at Linköping University, Sweden. Since 1993, he works at Inria, France, as a Research Scientist. His main research interests are in nonlinear system identification, fault diagnosis and biomedical signal processing. 